Communications in Applied Geometry.

ISSN 2249-4286 Volume 2, Number 1 (2012) pp. 7-23

(C) Research India Publications

http://www.ripublication.com/cag.htm

\title{
A Conjecture of Ducci Sequences
}

\author{
Euich Miztani, Akihiro Nozaki, and Toru Sawatari \\ JEIN Institute for Fundamental Science(JIFS) \\ VBL Kyoto University, Yoshida honmachi, \\ sakyo-ku, Kyoto 600-8813, Japan \\ Professor Emeritus, Otsuma Women's University \\ 2-7-1 Karakida, Tama-shi, Tokyo 206-8540, Japan \\ Microsoft Japan Co., Ltd \\ Shinagawa Grand Central Tower 2-16-3, \\ Konan Minato-ku, Tokyo 108-0075, Japan
}

\begin{abstract}
In this paper, we argue an arithmetic issue also known as Ducci's 4 or nnumber game. First we set an arbitrary non-negative integer at each vertex of a polygon, and apply the following procedure: at the center of each edge, set the difference of two integers at both ends of the edge, and connect the centers of adjacent edges: in this way, we obtain a new polygon with non-negative integers at every vertex. We apply the same procedure recursively, until all numbers set around the polygon become zeros. In the case the number $\mathrm{N}$ of edges of the polygon is four, it has been claimed that the procedure will always terminate in finite steps, and it is proved by B. Freedman [1]. For an arbitrary $\mathrm{N}$, we know the following facts.

1. If the number $\mathrm{N}$ of edges of the polygon is a power of two, the recursive procedure will always terminate in finite steps.

2. Otherwise, there exits some non-negative integers at vertices of the polygon, from which the recursive procedure will never terminate.

This time, we discuss the following fact: Let $\mathrm{A}, \mathrm{B}$, or $\mathrm{C}$ be positive integers on consecutive vertices on polygon $A \leq C \leq B, A \geq C \geq B, B \leq A \leq C$, or $\mathrm{B} \geq \mathrm{A} \geq \mathrm{C}$, the recursive procedure by the binary operation which vertices $\mathrm{N}$ are four will always terminate by four steps. In this paper, we prove it and also discuss further general case.
\end{abstract}


Keywords: Ducci sequences, polygon subtraction, recursive procedure, binary operation.

2010 Mathematics Subject Classification: 11A99.

\section{Introduction}

Historically, the arithmetic issue is well known as Ducci's 4 or n-number game. It can be arranged as follows. As shown in Fig.1, The arranged game sets non-negative integers at every corner of a square, and put at the center of each edge the difference of two integers at both ends of the edge. By connecting the centers of adjacent edges, he obtains a new square with non-negative integers at every corner. The procedure will be repeated recursively, until all numbers at corners become zeros. He noted that the procedure will always terminate in finite steps.

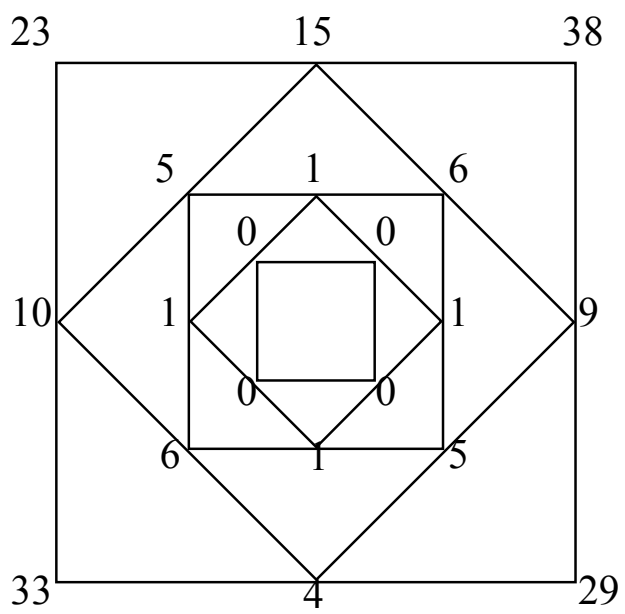

Figure 1

\section{Notations and Basic Concepts}

In what follows we give basic concepts and fundamental properties, in order to formulate our basic conjecture on the above mentioned problem.

Definition 2.1. Serializing any non-negative integers $a_{1}, a_{2}, a_{3}, \ldots, a_{n-1}, a_{N}$ for vertices of a polygon with $\mathrm{N}$ vertices, let $\left[\mathrm{a}_{\mathrm{n}-1}, \mathrm{a}_{\mathrm{n}}\right]$ and $\left[\mathrm{a}_{\mathrm{N}}, \mathrm{a}_{1}\right](\mathrm{N} \geq \mathrm{n})$ be absolute value of binary operation of the circular permutation by the recursive procedure noted above. Therefore, the equation of the recursive procedure is

$$
\left[a_{n-1}, a_{n}\right]=\left|a_{n-1}-a_{n}\right|,\left[a_{N}, a_{1}\right]=\left|a_{N}-a_{1}\right|
$$

Then, repeat the recursive procedure until the terminate values are zeros.

Remark: a couple of members in square brackets are commutative because they are positive integers. 
Lemma 2.1. Let $\mathrm{A}, \mathrm{B}$, and $\mathrm{C}$ be non-negative integers on consecutive vertices on polygon.

i). If $\mathrm{A}<\mathrm{B}<\mathrm{C}$,

$[[\mathrm{A}, \mathrm{B}],[\mathrm{B}, \mathrm{C}]]=[\mathrm{C},[\mathrm{A}, 2 \mathrm{~B}]]$

ii). If $A>B>C$,

$[[\mathrm{A}, \mathrm{B}],[\mathrm{B}, \mathrm{C}]]=[\mathrm{A},[\mathrm{C}, 2 \mathrm{~B}]]$

iii). If $A \leq C \leq B, A \geq C \geq B, B \leq A \leq C$, or $B \geq A \geq C$,

$[[\mathrm{A}, \mathrm{B}],[\mathrm{B}, \mathrm{C}]]=[\mathrm{A}, \mathrm{C}]$

Remark : Every combination of the six possible ordering of three positive integers A, $\mathrm{B}$, and $\mathrm{C}$ is treated in one of the three cases i), ii) or iii).

Proof.

i). If $\mathrm{A}<\mathrm{B}<\mathrm{C}$,

$[[\mathrm{A}, \mathrm{B}],[\mathrm{B}, \mathrm{C}]]=|(\mathrm{B}-\mathrm{A})-(\mathrm{C}-\mathrm{B})|=|(-\mathrm{A}+2 \mathrm{~B})-\mathrm{C}|=[\mathrm{C},[\mathrm{A}, 2 \mathrm{~B}]]$

ii). If $A>B>C$,

$[[\mathrm{A}, \mathrm{B}],[\mathrm{B}, \mathrm{C}]]=|(\mathrm{A}-\mathrm{B})-(\mathrm{B}-\mathrm{C})|=|\mathrm{A}-(2 \mathrm{~B}-\mathrm{C})|=[\mathrm{A},[\mathrm{C}, 2 \mathrm{~B}]]$

iii).

If $\mathrm{A} \leq \mathrm{C} \leq \mathrm{B}$,

$[[\mathrm{A}, \mathrm{B}],[\mathrm{B}, \mathrm{C}]]=(\mathrm{B}-\mathrm{A})-(\mathrm{B}-\mathrm{C})=\mathrm{C}-\mathrm{A}=[\mathrm{A}, \mathrm{C}]$

If $A \geq C \geq B$,

$[[A, B],[B, C]]=(A-B)-(C-B)=A-C=[A, C]$

If $B \leq A \leq C$,

$[[\mathrm{A}, \mathrm{B}],[\mathrm{B}, \mathrm{C}]]=(\mathrm{C}-\mathrm{B})-(\mathrm{A}-\mathrm{B})=\mathrm{C}-\mathrm{A}=[\mathrm{A}, \mathrm{C}]$

If $B \geq A \geq C$,

$[[\mathrm{A}, \mathrm{B}],[\mathrm{B}, \mathrm{C}]]=(\mathrm{B}-\mathrm{C})-(\mathrm{B}-\mathrm{A})=\mathrm{A}-\mathrm{C}=[\mathrm{A}, \mathrm{C}]$

Those case i), ii), and iii) are combined in the process of operations, but it is very difficult to research all the processes because of the complication. Therefore, let us check each of the cases separately.

Now, let us algorithmically verify positive integers set around all the vertices of square $(; \mathrm{N}=4)$ based on the lemma 2.1 .

i). If $\mathrm{A}<\mathrm{B}<\mathrm{C}$ for any consecutive $\mathrm{a}_{\mathrm{n}}$ and the following integers after recursive procedures except for cases of $a_{N-1}, a_{N}$, and $a_{1}\left(; a_{1}<a_{N-1}<a_{N}\right.$ by condition of the circular permutation, therefore it belongs to the case iii)) and $\mathrm{a}_{\mathrm{N}}, \mathrm{a}_{1}$, and $\mathrm{a}_{2}\left(; \mathrm{a}_{1}<\mathrm{a}_{2}<\right.$ $\mathrm{a}_{\mathrm{N}}$,

Step 1

$\left[a_{1}, a_{2}\right],\left[a_{2}, a_{3}\right],\left[a_{3}, a_{4}\right],\left[a_{4}, a_{1}\right]$

Step2

$\left[\left[a_{1}, a_{2}\right],\left[a_{2}, a_{3}\right]\right]=\left[a_{3},\left[a_{1}, 2 a_{2}\right]\right]$

$\left[\left[a_{2}, a_{3}\right],\left[a_{3}, a_{4}\right]\right]=\left[a_{4},\left[a_{2}, 2 a_{3}\right]\right]$

$\left[\left[a_{3}, a_{4}\right],\left[a_{4}, a_{1}\right]\right]=\left[a_{3}, a_{1}\right]$

$\left[\left[a_{4}, a_{1}\right],\left[a_{1}, a_{2}\right]\right]=\left[a_{4}, a_{2}\right]$

Step3

$\left[\left[\mathrm{a}_{3},\left[\mathrm{a}_{1}, 2 \mathrm{a}_{2}\right]\right],\left[\mathrm{a}_{4},\left[\mathrm{a}_{2}, 2 \mathrm{a}_{3}\right]\right]\right]$ 
$\left[\left[\mathrm{a}_{4},\left[\mathrm{a}_{2}, 2 \mathrm{a}_{3}\right]\right],\left[\mathrm{a}_{3}, \mathrm{a}_{1}\right]\right]$

$\left[\left[\mathrm{a}_{3}, \mathrm{a}_{1}\right],\left[\mathrm{a}_{4}, \mathrm{a}_{2}\right]\right]$

$\left[\left[\mathrm{a}_{4}, \mathrm{a}_{2}\right],\left[\mathrm{a}_{3},\left[\mathrm{a}_{1}, 2 \mathrm{a}_{2}\right]\right]\right]$

Step4

$\left[\left[\left[\mathrm{a}_{3},\left[\mathrm{a}_{1}, 2 \mathrm{a}_{2}\right]\right],\left[\mathrm{a}_{4},\left[\mathrm{a}_{2}, 2 \mathrm{a}_{3}\right]\right]\right],\left[\left[\mathrm{a}_{4},\left[\mathrm{a}_{2}, 2 \mathrm{a}_{3}\right]\right],\left[\mathrm{a}_{3}, \mathrm{a}_{1}\right]\right]\right]$

$\left[\left[\left[a_{4},\left[a_{2}, 2 a_{3}\right]\right],\left[a_{3}, a_{1}\right]\right],\left[\left[a_{3}, a_{1}\right],\left[a_{4}, a_{2}\right]\right]\right]$

$\left[\left[\left[\mathrm{a}_{3}, \mathrm{a}_{1}\right],\left[\mathrm{a}_{4}, \mathrm{a}_{2}\right]\right],\left[\left[\mathrm{a}_{4}, \mathrm{a}_{2}\right],\left[\mathrm{a}_{3},\left[\mathrm{a}_{1}, 2 \mathrm{a}_{2}\right]\right]\right]\right.$

$\left[\left[\left[a_{4}, a_{2}\right],\left[a_{3},\left[a_{1}, 2 a_{2}\right]\right]\right],\left[\left[a_{4}, a_{2}\right]\right],\left[a_{3},\left[a_{1}, 2 a_{2}\right]\right]\right]$

Since the values in square brackets diverge, we stop the operation.

ii). If $\mathrm{A}>\mathrm{B}>\mathrm{C}$ for any consecutive $\mathrm{a}_{\mathrm{n}}$ and the following integers after recursive procedures except for cases of $a_{N-1}, a_{N}, a_{1}$ and $a_{N}, a_{1}, a_{2}$,

then the results are the same as the case i) and we do not.

iii). If $A \leq C \leq B, A \geq C \geq B, B \leq A \leq C$, and $B \geq A \geq C$ for any consecutive $a_{n}$, Step 1

$\left[a_{1}, a_{2}\right],\left[a_{2}, a_{3}\right],\left[a_{3}, a_{4}\right],\left[a_{4}, a_{1}\right]$

Step2

$\left[\left[a_{1}, a_{2}\right],\left[a_{2}, a_{3}\right]\right]=\left[a_{1}, a_{3}\right]$

$\left[\left[a_{2}, a_{3}\right],\left[a_{3}, a_{4}\right]\right]=\left[a_{2}, a_{4}\right]$

$\left[\left[\mathrm{a}_{3}, \mathrm{a}_{4}\right],\left[\mathrm{a}_{4}, \mathrm{a}_{1}\right]\right]=\left[\mathrm{a}_{3}, \mathrm{a}_{1}\right]$

$\left[\left[a_{4}, a_{1}\right],\left[a_{1}, a_{2}\right]\right]=\left[a_{4}, a_{2}\right]$

Step3

$\left[\left[\mathrm{a}_{1}, \mathrm{a}_{3}\right],\left[\mathrm{a}_{2}, \mathrm{a}_{4}\right]\right]$

$\left[\left[a_{2}, a_{4}\right],\left[a_{3}, a_{1}\right]\right]=\left[\left[a_{1}, a_{3}\right],\left[a_{2}, a_{4}\right]\right]$

$\left[\left[a_{3}, a_{1}\right],\left[a_{4}, a_{2}\right]\right]=\left[\left[a_{1}, a_{3}\right],\left[a_{2}, a_{4}\right]\right]$

$\left[\left[a_{4}, a_{2}\right],\left[a_{1}, a_{3}\right]\right]=\left[\left[a_{1}, a_{3}\right],\left[a_{2}, a_{4}\right]\right]$

Step4

0 allout.

Therefore, we get the valid results for the case iii). As verified above, we assume that the terminal values based on the case iii) of lemma 2.1 will be zeros, only if the number of vertices is four. Then,

Conjecture. Let $A, B$, and $C$ be positive integers on consecutive vertices on polygon $A \leq C \leq B, A \geq C \geq B, B \leq A \leq C$, or $B \geq A \geq C$, the recursive procedure by the binary operation which the number of vertices is four will always terminate by four steps.

\section{Postscript.}

We were originally supposed to offer the conjecture wider as follows,

Conjecture. Let $A, B$, and $C$ be positive integers on consecutive vertices on polygon $A \leq C \leq B, A \geq C \geq B, B \leq A \leq C$, or $B \geq A \geq C$, the recursive procedure by the binary operation which the number of vertices is a power of two will always terminate by the power of two steps.

For example, under the special conditions, if $\mathrm{N}=2,4$, and 8 , then the operation terminates by 2,4 , and 8 steps as calculated above. 
To verify it above, let $\mathrm{A}, \mathrm{B}$, and $\mathrm{C}$ be positive integers on consecutive vertices on polygon and $A \leq C \leq B, A \geq C \geq B, B \leq A \leq C$, or $B \geq A \geq C$, and we calculate the differences to see if the results by recursive procedures are terminally zeros.

First of all, if $\mathrm{N}=2$,

Remark: a polygon with two vertices (and double sides).

Step1

$\left[\mathrm{a}_{1}, \mathrm{a}_{2}\right],\left[\mathrm{a}_{2}, \mathrm{a}_{1}\right]$

Step2

$\left[\left[a_{1}, a_{2}\right],\left[a_{2}, a_{1}\right]\right]=\left[a_{1}, a_{1}\right]=0$

$\mathrm{N}=3$,

Step1

$\left[a_{1}, a_{2}\right],\left[a_{2}, a_{3}\right],\left[a_{3}, a_{1}\right]$

Step2

$\left[\left[\mathrm{a}_{1}, \mathrm{a}_{2}\right],\left[\mathrm{a}_{2}, \mathrm{a}_{3}\right]\right]=\left[\mathrm{a}_{1}, \mathrm{a}_{3}\right]$

$\left[\left[a_{2}, a_{3}\right],\left[a_{3}, a_{1}\right]\right]=\left[a_{2}, a_{1}\right]$

$\left[\left[a_{3}, a_{1}\right],\left[a_{1}, a_{2}\right]\right]=\left[a_{3}, a_{2}\right]$

Step3

$\left[\left[a_{1}, a_{3}\right],\left[a_{2}, a_{1}\right]\right]$

$\left[\left[\mathrm{a}_{2}, \mathrm{a}_{1}\right],\left[\mathrm{a}_{3}, \mathrm{a}_{2}\right]\right]$

$\left[\left[\mathrm{a}_{3}, \mathrm{a}_{2}\right],\left[\mathrm{a}_{1}, \mathrm{a}_{3}\right]\right]$

Step4 (Remark: any integer at vertices (, not only any initial integer before recursive procedure) follows the case iii) of lemma 2.1. )

$\left[\left[\left[a_{1}, a_{3}\right],\left[a_{2}, a_{1}\right]\right],\left[\left[a_{2}, a_{1}\right],\left[a_{3}, a_{2}\right]\right]\right]\left(=\left[A^{\prime}, B^{\prime}\right] .\left[B^{\prime}, C^{\prime}\right]\right)$

$=\left[\left[a_{1}, a_{3}\right],\left[a_{3}, a_{2}\right]\right](=[[A, B],[B, C]])=\left[\left[a_{1}, a_{2}\right]\right.$

$\left[\left[\left[a_{2}, a_{1}\right],\left[a_{3}, a_{2}\right]\right],\left[\left[a_{3}, a_{2}\right],\left[a_{1}, a_{3}\right]\right]\right]=\left[a_{2}, a_{3}\right]$

$\left[\left[\left[a_{3}, a_{2}\right],\left[a_{1}, a_{3}\right]\right],\left[\left[a_{1}, a_{3}\right],\left[a_{2}, a_{1}\right]\right]\right]=\left[a_{3}, a_{1}\right]$

Step5

Return to Step2; the values are never zeros.

$\mathrm{N}=4$, it is as calculated above.

$\mathrm{N}=5$,

Step1

$\left[a_{1}, a_{2}\right],\left[a_{2}, a_{3}\right],\left[a_{3}, a_{4}\right],\left[a_{4}, a_{5}\right],\left[a_{5}, a_{1}\right]$

Step2

$\left[\left[a_{1}, a_{2}\right],\left[a_{2}, a_{3}\right]\right]=\left[a_{1}, a_{3}\right]$

$\left[\left[a_{2}, a_{3}\right],\left[a_{3}, a_{4}\right]\right]=\left[a_{2}, a_{4}\right]$

$\left[\left[a_{3}, a_{4}\right],\left[a_{4}, a_{5}\right]\right]=\left[a_{3}, a_{5}\right]$

$\left[\left[a_{4}, a_{5}\right],\left[a_{5}, a_{1}\right]\right]=\left[a_{4}, a_{1}\right]$

$\left[\left[a_{5}, a_{1}\right],\left[a_{1}, a_{2}\right]\right]=\left[a_{5}, a_{2}\right]$

Step3

$\left[\left[a_{1}, a_{3}\right],\left[a_{2}, a_{4}\right]\right]$

$\left[\left[a_{2}, a_{4}\right],\left[a_{3}, a_{5}\right]\right]$ 
$\left[\left[a_{3}, a_{5}\right],\left[a_{4}, a_{1}\right]\right]$

$\left[\left[\mathrm{a}_{4}, \mathrm{a}_{1}\right],\left[\mathrm{a}_{5}, \mathrm{a}_{2}\right]\right]$

$\left[\left[\mathrm{a}_{5}, \mathrm{a}_{2}\right],\left[\mathrm{a}_{1}, \mathrm{a}_{3}\right]\right]$

Step4 (Remark: any integer at vertices follows the case iii) of lemma 2.1.)

$\left[\left[\left[\mathrm{a}_{1}, \mathrm{a}_{3}\right],\left[\mathrm{a}_{2}, \mathrm{a}_{4}\right]\right],\left[\left[\mathrm{a}_{2}, \mathrm{a}_{4}\right],\left[\mathrm{a}_{3}, \mathrm{a}_{5}\right]\right]\right]\left(=\left[\left[\mathrm{A}^{\prime}, \mathrm{B}^{\prime}\right],\left[\mathrm{B}^{\prime}, \mathrm{C}^{\prime}\right]\right]=\left[\mathrm{A}^{\prime}, \mathrm{C}^{\prime}\right]\right)$

$=\left[\left[a_{1}, a_{3}\right],\left[a_{3}, a_{5}\right]\right](=[[A, B],[B, C]])=\left[a_{1}, a_{5}\right]=\left[a_{5}, a_{1}\right]$

$\left[\left[\left[a_{2}, a_{4}\right],\left[a_{3}, a_{5}\right]\right],\left[\left[a_{3}, a_{5}\right],\left[a_{4}, a_{1}\right]\right]\right]=\left[\left[a_{2}, a_{4}\right],\left[a_{4}, a_{1}\right]\right]=\left[a_{2}, a_{1}\right]=\left[a_{1}, a_{2}\right]$

$\left[\left[\left[a_{3}, a_{5}\right],\left[a_{4}, a_{1}\right]\right],\left[\left[a_{4}, a_{1}\right],\left[a_{5}, a_{2}\right]\right]\right]=\left[\left[a_{3}, a_{5}\right],\left[a_{5}, a_{2}\right]\right]=\left[a_{3}, a_{2}\right]=\left[a_{2}, a_{3}\right]$

$\left[\left[\left[a_{4}, a_{1}\right],\left[a_{5}, a_{2}\right]\right],\left[\left[a_{5}, a_{2}\right],\left[a_{1}, a_{3}\right]\right]\right]=\left[\left[a_{4}, a_{1}\right],\left[a_{1}, a_{3}\right]\right]=\left[a_{4}, a_{3}\right]=\left[a_{3}, a_{4}\right]$

$\left[\left[\left[a_{5}, a_{2}\right],\left[a_{1}, a_{3}\right]\right],\left[\left[a_{1}, a_{3}\right],\left[a_{2}, a_{4}\right]\right]\right]=\left[\left[a_{5}, a_{2}\right],\left[a_{2}, a_{4}\right]\right]=\left[a_{5}, a_{4}\right]=\left[a_{4}, a_{5}\right]$

Step5

Return to Step2

$\mathrm{N}=6$,

Step1

$\left[a_{1}, a_{2}\right],\left[a_{2}, a_{3}\right],\left[a_{3}, a_{4}\right],\left[a_{4}, a_{5}\right],\left[a_{5}, a_{6}\right],\left[a_{6}, a_{1}\right]$

Step2

$\left[\left[a_{1}, a_{2}\right],\left[a_{2}, a_{3}\right]\right]=\left[a_{1}, a_{3}\right]$

$\left[\left[a_{2}, a_{3}\right],\left[a_{3}, a_{4}\right]\right]=\left[a_{2}, a_{4}\right]$

$\left[\left[a_{3}, a_{4}\right],\left[a_{4}, a_{5}\right]\right]=\left[a_{3}, a_{5}\right]$

$\left[\left[a_{4}, a_{5}\right],\left[a_{5}, a_{6}\right]\right]=\left[a_{4}, a_{6}\right]$

$\left[\left[\mathrm{a}_{5}, \mathrm{a}_{6}\right],\left[\mathrm{a}_{6}, \mathrm{a}_{1}\right]\right]=\left[\mathrm{a}_{5}, \mathrm{a}_{1}\right]$

$\left[\left[a_{6}, a_{1}\right],\left[a_{1}, a_{2}\right]\right]=\left[a_{6}, a_{2}\right]$

Step3

$\left[\left[a_{1}, a_{3}\right],\left[a_{2}, a_{4}\right]\right]$

$\left[\left[a_{2}, a_{4}\right],\left[a_{3}, a_{5}\right]\right]$

$\left[\left[a_{3}, a_{5}\right],\left[a_{4}, a_{6}\right]\right]$

$\left[\left[a_{4}, a_{6}\right],\left[a_{5}, a_{1}\right]\right]$

$\left[\left[a_{5}, a_{1}\right],\left[a_{6}, a_{2}\right]\right]$

$\left[\left[\mathrm{a}_{6}, \mathrm{a}_{2}\right],\left[\mathrm{a}_{1}, \mathrm{a}_{3}\right]\right]$

Step4 (Remark: any integer at vertices follows the case iii) of lemma 2.1.)

$\left[\left[\left[a_{1}, a_{3}\right],\left[a_{2}, a_{4}\right]\right],\left[\left[a_{2}, a_{4}\right],\left[a_{3}, a_{5}\right]\right]\right]=\left[\left[a_{1}, a_{3}\right],\left[a_{3}, a_{5}\right]\right]=\left[a_{1}, a_{5}\right]=\left[a_{5}, a_{1}\right]$

$\left[\left[\left[a_{2}, a_{4}\right],\left[a_{3}, a_{5}\right]\right],\left[\left[a_{3}, a_{5}\right],\left[a_{4}, a_{6}\right]\right]\right]=\left[\left[a_{2}, a_{4}\right],\left[a_{4}, a_{6}\right]\right]=\left[a_{2}, a_{6}\right]=\left[a_{6}, a_{2}\right]$

$\left[\left[\left[a_{3}, a_{5}\right],\left[a_{4}, a_{6}\right]\right],\left[\left[a_{4}, a_{6}\right],\left[a_{5}, a_{1}\right]\right]\right]=\left[\left[a_{3}, a_{5}\right],\left[a_{5}, a_{1}\right]\right]=\left[a_{3}, a_{1}\right]=\left[a_{1}, a_{3}\right]$

$\left[\left[\left[a_{4}, a_{6}\right],\left[a_{5}, a_{1}\right]\right],\left[\left[a_{5}, a_{1}\right],\left[a_{6}, a_{2}\right]\right]\right]=\left[\left[a_{4}, a_{6}\right],\left[a_{6}, a_{2}\right]\right]=\left[a_{4}, a_{2}\right]=\left[a_{2}, a_{4}\right]$

$\left[\left[\left[a_{5}, a_{1}\right],\left[a_{6}, a_{2}\right]\right],\left[\left[a_{6}, a_{2}\right],\left[a_{1}, a_{3}\right]\right]\right]=\left[\left[a_{5}, a_{1}\right],\left[a_{1}, a_{3}\right]\right]=\left[a_{5}, a_{3}\right]=\left[a_{3}, a_{5}\right]$

$\left[\left[\left[a_{6}, a_{2}\right],\left[a_{1}, a_{3}\right]\right],\left[\left[a_{1}, a_{3}\right],\left[a_{2}, a_{4}\right]\right]\right]=\left[\left[a_{6}, a_{2}\right],\left[a_{2}, a_{4}\right]\right]=\left[a_{6}, a_{4}\right]=\left[a_{4}, a_{6}\right]$

Return to Step3.

$\mathrm{N}=7$,

Step1

$\left[a_{1}, a_{2}\right],\left[a_{2}, a_{3}\right],\left[a_{3}, a_{4}\right],\left[a_{4}, a_{5}\right],\left[a_{5}, a_{6}\right],\left[a_{6}, a_{7}\right],\left[a_{7}, a_{1}\right]$

Step2

$\left[\left[\mathrm{a}_{1}, \mathrm{a}_{2}\right],\left[\mathrm{a}_{2}, \mathrm{a}_{3}\right]\right]=\left[\mathrm{a}_{1}, \mathrm{a}_{3}\right]$

$\left[\left[a_{2}, a_{3}\right],\left[a_{3}, a_{4}\right]\right]=\left[a_{2}, a_{4}\right]$ 
$\left[\left[a_{3}, a_{4}\right],\left[a_{4}, a_{5}\right]\right]=\left[a_{3}, a_{5}\right]$
$\left[\left[a_{4}, a_{5}\right],\left[a_{5}, a_{6}\right]\right]=\left[a_{4}, a_{6}\right]$
$\left[\left[a_{5}, a_{6}\right],\left[a_{6}, a_{7}\right]\right]=\left[a_{5}, a_{7}\right]$
$\left[\left[a_{6}, a_{7}\right],\left[a_{7}, a_{1}\right]\right]=\left[a_{6}, a_{1}\right]$
$\left[\left[a_{7}, a_{1}\right],\left[a_{1}, a_{2}\right]\right]=\left[a_{7}, a_{2}\right]$

Step3

$\left[\left[a_{1}, a_{3}\right],\left[a_{2}, a_{4}\right]\right]$

$\left[\left[a_{2}, a_{4}\right],\left[a_{3}, a_{5}\right]\right]$

$\left[\left[a_{3}, a_{5}\right],\left[a_{4}, a_{6}\right]\right]$

$\left[\left[a_{4}, a_{6}\right],\left[a_{5}, a_{7}\right]\right]$

$\left[\left[a_{5}, a_{7}\right],\left[a_{6}, a_{1}\right]\right]$

$\left[\left[a_{6}, a_{1}\right],\left[a_{7}, a_{2}\right]\right]$

$\left[\left[a_{7}, a_{2}\right],\left[a_{1}, a_{3}\right]\right]$

Step4 (Remark: any integer at vertices follows the case iii) of lemma 2.1.)

$\left[\left[\left[\mathrm{a}_{1}, \mathrm{a}_{3}\right],\left[\mathrm{a}_{2}, \mathrm{a}_{4}\right]\right],\left[\left[\mathrm{a}_{2}, \mathrm{a}_{4}\right],\left[\mathrm{a}_{3}, \mathrm{a}_{5}\right]\right]\right]=\left[\left[\mathrm{a}_{1}, \mathrm{a}_{3}\right],\left[\mathrm{a}_{3}, \mathrm{a}_{5}\right]\right]=\left[\mathrm{a}_{1}, \mathrm{a}_{5}\right]$

$\left[\left[\left[a_{2}, a_{4}\right],\left[a_{3}, a_{5}\right]\right],\left[\left[a_{3}, a_{5}\right],\left[a_{4}, a_{6}\right]\right]\right]=\left[\left[a_{2}, a_{4}\right],\left[a_{4}, a_{6}\right]\right]=\left[a_{2}, a_{6}\right]$

$\left[\left[\left[a_{3}, a_{5}\right],\left[a_{4}, a_{6}\right]\right],\left[\left[a_{4}, a_{6}\right],\left[a_{5}, a_{7}\right]\right]\right]=\left[\left[a_{3}, a_{5}\right],\left[a_{5}, a_{7}\right]\right]=\left[a_{3}, a_{7}\right]$

$\left[\left[\left[a_{4}, a_{6}\right],\left[a_{5}, a_{7}\right]\right],\left[\left[a_{5}, a_{7}\right],\left[a_{6}, a_{1}\right]\right]\right]=\left[\left[a_{4}, a_{6}\right],\left[a_{6}, a_{1}\right]\right]=\left[a_{4}, a_{1}\right]$

$\left[\left[\left[a_{5}, a_{7}\right],\left[a_{6}, a_{1}\right]\right],\left[\left[a_{6}, a_{1}\right],\left[a_{7}, a_{2}\right]\right]\right]=\left[\left[a_{5}, a_{7}\right],\left[a_{7}, a_{2}\right]\right]=\left[a_{5}, a_{2}\right]$

$\left[\left[\left[a_{6}, a_{1}\right],\left[a_{7}, a_{2}\right]\right],\left[\left[a_{7}, a_{2}\right],\left[a_{1}, a_{3}\right]\right]\right]=\left[\left[a_{6}, a_{1}\right],\left[a_{1}, a_{3}\right]\right]=\left[a_{6}, a_{3}\right]$

$\left[\left[\left[a_{7}, a_{2}\right],\left[a_{1}, a_{3}\right]\right],\left[\left[a_{1}, a_{3}\right],\left[a_{2}, a_{4}\right]\right]\right]=\left[\left[a_{7}, a_{2}\right],\left[a_{2}, a_{4}\right]\right]=\left[a_{7}, a_{4}\right]$

\section{Step5}

$\left[\left[a_{1}, a_{5}\right],\left[a_{2}, a_{6}\right]\right]$

$\left[\left[\mathrm{a}_{2}, \mathrm{a}_{6}\right],\left[\mathrm{a}_{3}, \mathrm{a}_{7}\right]\right]$

$\left[\left[a_{3}, a_{7}\right],\left[a_{4}, a_{1}\right]\right]$

$\left[\left[a_{4}, a_{1}\right],\left[a_{5}, a_{2}\right]\right]$

$\left[\left[a_{5}, a_{2}\right],\left[a_{6}, a_{3}\right]\right]$

$\left[\left[a_{6}, a_{3}\right],\left[a_{7}, a_{4}\right]\right]$

$\left[\left[a_{7}, a_{4}\right],\left[a_{1}, a_{5}\right]\right]$

Step6 (Remark: any integer at vertices follows the case iii) of lemma 2.1.)

$\left[\left[\left[a_{1}, a_{5}\right],\left[a_{2}, a_{6}\right]\right],\left[\left[a_{2}, a_{6}\right],\left[a_{3}, a_{7}\right]\right]\right]=\left[\left[a_{1}, a_{5}\right],\left[a_{3}, a_{7}\right]\right]$

$\left[\left[\left[a_{2}, a_{6}\right],\left[a_{3}, a_{7}\right]\right],\left[\left[a_{3}, a_{7}\right],\left[a_{4}, a_{1}\right]\right]\right]=\left[\left[a_{2}, a_{6}\right],\left[a_{4}, a_{1}\right]\right]$

$\left[\left[\left[a_{3}, a_{7}\right],\left[a_{4}, a_{1}\right]\right],\left[\left[a_{4}, a_{1}\right],\left[a_{5}, a_{2}\right]\right]\right]=\left[\left[a_{3}, a_{7}\right],\left[a_{5}, a_{2}\right]\right]$

$\left[\left[\left[a_{4}, a_{1}\right],\left[a_{5}, a_{2}\right]\right],\left[\left[a_{5}, a_{2}\right],\left[a_{6}, a_{3}\right]\right]\right]=\left[\left[a_{4}, a_{1}\right],\left[a_{6}, a_{3}\right]\right]$

$\left[\left[\left[a_{5}, a_{2}\right],\left[a_{6}, a_{3}\right]\right],\left[\left[a_{6}, a_{3}\right],\left[a_{7}, a_{4}\right]\right]\right]=\left[\left[a_{5}, a_{2}\right],\left[a_{7}, a_{4}\right]\right]$

$\left[\left[\left[a_{6}, a_{3}\right],\left[a_{7}, a_{4}\right]\right],\left[\left[a_{7}, a_{4}\right],\left[a_{1}, a_{5}\right]\right]\right]=\left[\left[a_{6}, a_{3}\right],\left[a_{1}, a_{5}\right]\right]$

$\left[\left[\left[a_{7}, a_{4}\right],\left[a_{1}, a_{5}\right]\right],\left[\left[a_{1}, a_{5}\right],\left[a_{2}, a_{6}\right]\right]\right]=\left[\left[a_{7}, a_{4}\right],\left[a_{2}, a_{6}\right]\right]$

Step7

$\left[\left[\left[a_{1}, a_{5}\right],\left[a_{3}, a_{7}\right]\right],\left[\left[a_{2}, a_{6}\right],\left[a_{4}, a_{1}\right]\right]\right]$

$\left[\left[\left[\mathrm{a}_{2}, \mathrm{a}_{6}\right],\left[\mathrm{a}_{4}, \mathrm{a}_{1}\right]\right],\left[\left[\mathrm{a}_{3}, \mathrm{a}_{7}\right],\left[\mathrm{a}_{5}, \mathrm{a}_{2}\right]\right]\right]$

$\left[\left[\left[a_{3}, a_{7}\right],\left[a_{5}, a_{2}\right]\right],\left[\left[a_{4}, a_{1}\right],\left[a_{6}, a_{3}\right]\right]\right]$

$\left[\left[\left[a_{4}, a_{1}\right],\left[a_{6}, a_{3}\right]\right],\left[\left[a_{5}, a_{2}\right],\left[a_{7}, a_{4}\right]\right]\right]$

$\left[\left[\left[a_{5}, a_{2}\right],\left[a_{7}, a_{4}\right]\right],\left[\left[a_{6}, a_{3}\right],\left[a_{1}, a_{5}\right]\right]\right]$

$\left[\left[\left[a_{6}, a_{3}\right],\left[a_{1}, a_{5}\right]\right],\left[\left[a_{7}, a_{4}\right],\left[a_{2}, a_{6}\right]\right]\right]$

$\left[\left[\left[a_{7}, a_{4}\right],\left[a_{2}, a_{6}\right]\right],\left[\left[a_{1}, a_{5}\right],\left[a_{3}, a_{7}\right]\right]\right]$ 
Step8 (Remark: any integer at vertices follows the case iii) of lemma 2.1.)

$\left[\left[\left[\left[a_{1}, a_{5}\right],\left[a_{3}, a_{7}\right]\right],\left[\left[a_{2}, a_{6}\right],\left[a_{4}, a_{1}\right]\right]\right],\left[\left[\left[a_{2}, a_{6}\right],\left[a_{4}, a_{1}\right]\right],\left[\left[a_{3}, a_{7}\right],\left[a_{5}, a_{2}\right]\right]\right]\right]=\left[a_{1}, a_{2}\right]$ $\left[\left[\left[\left[a_{2}, a_{6}\right],\left[a_{4}, a_{1}\right]\right],\left[\left[a_{3}, a_{7}\right],\left[a_{5}, a_{2}\right]\right]\right],\left[\left[\left[a_{3}, a_{7}\right],\left[a_{5}, a_{2}\right]\right],\left[\left[a_{4}, a_{1}\right],\left[a_{6}, a_{3}\right]\right]\right]\right]=\left[a_{2}, a_{3}\right]$ $\left[\left[\left[\left[a_{3}, a_{7}\right],\left[a_{5}, a_{2}\right]\right],\left[\left[a_{4}, a_{1}\right],\left[a_{6}, a_{3}\right]\right]\right],\left[\left[\left[a_{4}, a_{1}\right],\left[a_{6}, a_{3}\right]\right],\left[\left[a_{5}, a_{2}\right],\left[a_{7}, a_{4}\right]\right]\right]\right]=\left[a_{3}, a_{4}\right]$ $\left[\left[\left[\left[a_{4}, a_{1}\right],\left[a_{6}, a_{3}\right]\right],\left[\left[a_{5}, a_{2}\right],\left[a_{7}, a_{4}\right]\right]\right],\left[\left[\left[a_{5}, a_{2}\right],\left[a_{7}, a_{4}\right]\right],\left[\left[a_{6}, a_{3}\right],\left[a_{1}, a_{5}\right]\right]\right]\right]=\left[a_{4}, a_{5}\right]$ $\left[\left[\left[\left[a_{5}, a_{2}\right],\left[a_{7}, a_{4}\right]\right],\left[\left[a_{6}, a_{3}\right],\left[a_{1}, a_{5}\right]\right]\right],\left[\left[\left[a_{6}, a_{3}\right],\left[a_{1}, a_{5}\right]\right],\left[\left[a_{7}, a_{4}\right],\left[a_{2}, a_{6}\right]\right]\right]\right]=\left[a_{5}, a_{6}\right]$ $\left[\left[\left[\left[a_{6}, a_{3}\right],\left[a_{1}, a_{5}\right]\right],\left[\left[a_{7}, a_{4}\right],\left[a_{2}, a_{6}\right]\right]\right],\left[\left[\left[a_{7}, a_{4}\right],\left[a_{2}, a_{6}\right]\right],\left[\left[a_{1}, a_{5}\right],\left[a_{3}, a_{7}\right]\right]\right]\right]=\left[a_{6}, a_{7}\right]$ $\left[\left[\left[\left[a_{7}, a_{4}\right],\left[a_{2}, a_{6}\right]\right],\left[\left[a_{1}, a_{5}\right],\left[a_{3}, a_{7}\right]\right]\right],\left[\left[\left[a_{1}, a_{5}\right],\left[a_{3}, a_{7}\right]\right],\left[\left[a_{2}, a_{6}\right],\left[a_{4}, a_{1}\right]\right]\right]\right]=\left[a_{7}, a_{1}\right]$ Step9

Return to Step2.

$\mathrm{N}=8$

Step1

$\left[a_{1}, a_{2}\right],\left[a_{2}, a_{3}\right],\left[a_{3}, a_{4}\right],\left[a_{4}, a_{5}\right],\left[a_{5}, a_{6}\right],\left[a_{6}, a_{7}\right],\left[a_{7}, a_{8}\right],\left[a_{8}, a_{1}\right]$

Step2

$\left[\left[a_{1}, a_{2}\right],\left[a_{2}, a_{3}\right]\right]=\left[a_{1}, a_{3}\right]$

$\left[\left[a_{2}, a_{3}\right],\left[a_{3}, a_{4}\right]\right]=\left[a_{2}, a_{4}\right]$

$\left[\left[a_{3}, a_{4}\right],\left[a_{4}, a_{5}\right]\right]=\left[a_{3}, a_{5}\right]$

$\left[\left[a_{4}, a_{5}\right],\left[a_{5}, a_{6}\right]\right]=\left[a_{4}, a_{6}\right]$

$\left[\left[a_{5}, a_{6}\right],\left[a_{6}, a_{7}\right]\right]=\left[a_{5}, a_{7}\right]$

$\left[\left[a_{6}, a_{7}\right],\left[a_{7}, a_{8}\right]\right]=\left[a_{6}, a_{8}\right]$

$\left[\left[a_{7}, a_{8}\right],\left[a_{8}, a_{1}\right]\right]=\left[a_{7}, a_{1}\right]$

$\left[\left[a_{8}, a_{1}\right],\left[a_{1}, a_{2}\right]\right]=\left[a_{8}, a_{2}\right]$

Step3

[[a $\left.\left.a_{1}, a_{3}\right],\left[a_{2}, a_{4}\right]\right]$

$\left[\left[a_{2}, a_{4}\right],\left[a_{3}, a_{5}\right]\right]$

$\left[\left[a_{3}, a_{5}\right],\left[a_{4}, a_{6}\right]\right]$

$\left[\left[a_{4}, a_{6}\right],\left[a_{5}, a_{7}\right]\right]$

$\left[\left[a_{5}, a_{7}\right],\left[a_{6}, a_{8}\right]\right]$

$\left[\left[a_{6}, a_{8}\right],\left[a_{7}, a_{1}\right]\right]$

$\left[\left[a_{7}, a_{1}\right],\left[a_{8}, a_{2}\right]\right]$

$\left[\left[a_{8}, a_{2}\right],\left[a_{1}, a_{3}\right]\right]$

Step4 (Remark: any integer at vertices follows the case iii) of lemma 2.1.)

$\left[\left[\left[a_{1}, a_{3}\right],\left[a_{2}, a_{4}\right]\right],\left[\left[a_{2}, a_{4}\right],\left[a_{3}, a_{5}\right]\right]\right]=\left[\left[a_{1}, a_{3}\right],\left[a_{3}, a_{5}\right]\right]=\left[a_{1}, a_{5}\right]$

$\left[\left[\left[a_{2}, a_{4}\right],\left[a_{3}, a_{5}\right]\right],\left[\left[a_{3}, a_{5}\right],\left[a_{4}, a_{6}\right]\right]\right]=\left[\left[a_{2}, a_{4}\right],\left[a_{4}, a_{6}\right]\right]=\left[a_{2}, a_{6}\right]$

$\left[\left[\left[a_{3}, a_{5}\right],\left[a_{4}, a_{6}\right]\right],\left[\left[a_{4}, a_{6}\right],\left[a_{5}, a_{7}\right]\right]\right]=\left[\left[a_{3}, a_{5}\right],\left[a_{5}, a_{7}\right]\right]=\left[a_{3}, a_{7}\right]$

$\left[\left[\left[a_{4}, a_{6}\right],\left[a_{5}, a_{7}\right]\right],\left[\left[a_{5}, a_{7}\right],\left[a_{6}, a_{8}\right]\right]\right]=\left[\left[a_{4}, a_{6}\right],\left[a_{6}, a_{8}\right]\right]=\left[a_{4}, a_{8}\right]$

$\left[\left[\left[a_{5}, a_{7}\right],\left[a_{6}, a_{8}\right]\right],\left[\left[a_{6}, a_{8}\right],\left[a_{7}, a_{1}\right]\right]\right]=\left[\left[a_{5}, a_{7}\right],\left[a_{7}, a_{1}\right]\right]=\left[a_{5}, a_{1}\right]$

$\left[\left[\left[a_{6}, a_{8}\right],\left[a_{7}, a_{1}\right]\right],\left[\left[a_{7}, a_{1}\right],\left[a_{8}, a_{2}\right]\right]\right]=\left[\left[a_{6}, a_{8}\right],\left[a_{8}, a_{2}\right]\right]=\left[a_{6}, a_{2}\right]$

$\left[\left[\left[a_{7}, a_{1}\right],\left[a_{8}, a_{2}\right]\right],\left[\left[a_{8}, a_{2}\right],\left[a_{1}, a_{3}\right]\right]\right]=\left[\left[a_{7}, a_{1}\right],\left[a_{1}, a_{3}\right]\right]=\left[a_{7}, a_{3}\right]$

$\left[\left[\left[a_{8}, a_{2}\right],\left[a_{1}, a_{3}\right]\right],\left[\left[a_{1}, a_{3}\right],\left[a_{2}, a_{4}\right]\right]\right]=\left[\left[a_{8}, a_{2}\right],\left[a_{2}, a_{4}\right]\right]=\left[a_{8}, a_{4}\right]$

\section{Step5}

$\left[\left[a_{1}, a_{5}\right],\left[a_{2}, a_{6}\right]\right]$

$\left[\left[a_{2}, a_{6}\right],\left[a_{3}, a_{7}\right]\right]$

$\left[\left[a_{3}, a_{7}\right],\left[a_{4}, a_{8}\right]\right]$ 
$\left[\left[a_{4}, a_{8}\right],\left[a_{5}, a_{1}\right]\right]$
$\left[\left[a_{5}, a_{1}\right],\left[a_{6}, a_{2}\right]\right]$
$\left[\left[a_{6}, a_{2}\right],\left[a_{7}, a_{3}\right]\right]$
$\left[\left[a_{7}, a_{3}\right],\left[a_{8}, a_{4}\right]\right]$
$\left[\left[a_{8}, a_{4}\right],\left[a_{1}, a_{5}\right]\right]$

Step6 (Remark: any integer at vertices follows the case iii) of lemma 2.1.)

$\left[\left[\left[a_{1}, a_{5}\right],\left[a_{2}, a_{6}\right]\right],\left[\left[a_{2}, a_{6}\right],\left[a_{3}, a_{7}\right]\right]\right]=\left[\left[a_{1}, a_{5}\right],\left[a_{3}, a_{7}\right]\right]$

$\left[\left[\left[a_{2}, a_{6}\right],\left[a_{3}, a_{7}\right]\right],\left[\left[a_{3}, a_{7}\right],\left[a_{4}, a_{8}\right]\right]\right]=\left[\left[a_{2}, a_{6}\right],\left[a_{4}, a_{8}\right]\right]$

$\left[\left[\left[\mathrm{a}_{3}, \mathrm{a}_{7}\right],\left[\mathrm{a}_{4}, \mathrm{a}_{8}\right]\right],\left[\left[\mathrm{a}_{4}, \mathrm{a}_{8}\right],\left[\mathrm{a}_{5}, \mathrm{a}_{1}\right]\right]\right]=\left[\left[\mathrm{a}_{3}, \mathrm{a}_{7}\right],\left[\mathrm{a}_{5}, \mathrm{a}_{1}\right]\right]$

$\left[\left[\left[\mathrm{a}_{4}, \mathrm{a}_{8}\right],\left[\mathrm{a}_{5}, \mathrm{a}_{1}\right]\right],\left[\left[\mathrm{a}_{5}, \mathrm{a}_{1}\right],\left[\mathrm{a}_{6}, \mathrm{a}_{2}\right]\right]\right]=\left[\left[\mathrm{a}_{4}, \mathrm{a}_{8}\right],\left[\mathrm{a}_{6}, \mathrm{a}_{2}\right]\right]$

$\left[\left[\left[a_{5}, a_{1}\right],\left[a_{6}, a_{2}\right]\right],\left[\left[a_{6}, a_{2}\right],\left[a_{7}, a_{3}\right]\right]\right]=\left[\left[a_{5}, a_{1}\right],\left[a_{7}, a_{3}\right]\right]$

$\left[\left[\left[a_{6}, a_{2}\right],\left[a_{7}, a_{3}\right]\right],\left[\left[a_{7}, a_{3}\right],\left[a_{8}, a_{4}\right]\right]\right]=\left[\left[a_{6}, a_{2}\right],\left[a_{8}, a_{4}\right]\right]$

$\left[\left[\left[a_{7}, a_{3}\right],\left[a_{8}, a_{4}\right]\right],\left[\left[a_{8}, a_{4}\right],\left[a_{1}, a_{5}\right]\right]\right]=\left[\left[a_{7}, a_{3}\right],\left[a_{1}, a_{5}\right]\right]$

$\left[\left[\left[a_{8}, a_{4}\right],\left[a_{1}, a_{5}\right]\right],\left[\left[a_{1}, a_{5}\right],\left[a_{2}, a_{6}\right]\right]\right]=\left[\left[a_{8}, a_{4}\right],\left[a_{2}, a_{6}\right]\right]$

Step7

$\left[\left[\left[a_{1}, a_{5}\right],\left[a_{3}, a_{7}\right]\right],\left[\left[a_{2}, a_{6}\right],\left[a_{4}, a_{8}\right]\right]\right]$

$\left[\left[\left[\mathrm{a}_{2}, \mathrm{a}_{6}\right],\left[\mathrm{a}_{4}, \mathrm{a}_{8}\right]\right],\left[\left[\mathrm{a}_{3}, \mathrm{a}_{7}\right],\left[\mathrm{a}_{5}, \mathrm{a}_{1}\right]\right]\right]=\left[\left[\left[\mathrm{a}_{1}, \mathrm{a}_{5}\right],\left[\mathrm{a}_{3}, \mathrm{a}_{7}\right]\right],\left[\left[\mathrm{a}_{2}, \mathrm{a}_{6}\right],\left[\mathrm{a}_{4}, \mathrm{a}_{8}\right]\right]\right]$

$\left[\left[\left[a_{3}, a_{7}\right],\left[a_{5}, a_{1}\right]\right],\left[\left[a_{4}, a_{8}\right],\left[a_{6}, a_{2}\right]\right]\right]=\left[\left[\left[a_{1}, a_{5}\right],\left[a_{3}, a_{7}\right]\right],\left[\left[a_{2}, a_{6}\right],\left[a_{4}, a_{8}\right]\right]\right]$

$\left[\left[\left[a_{4}, a_{8}\right],\left[a_{6}, a_{2}\right]\right],\left[\left[a_{5}, a_{1}\right],\left[a_{7}, a_{3}\right]\right]\right]=\left[\left[\left[a_{1}, a_{5}\right],\left[a_{3}, a_{7}\right]\right],\left[\left[a_{2}, a_{6}\right],\left[a_{4}, a_{8}\right]\right]\right]$

$\left[\left[\left[a_{5}, a_{1}\right],\left[a_{7}, a_{3}\right]\right],\left[\left[a_{6}, a_{2}\right],\left[a_{8}, a_{4}\right]\right]\right]=\left[\left[\left[a_{1}, a_{5}\right],\left[a_{3}, a_{7}\right]\right],\left[\left[a_{2}, a_{6}\right],\left[a_{4}, a_{8}\right]\right]\right]$

$\left[\left[\left[\mathrm{a}_{6}, \mathrm{a}_{2}\right],\left[\mathrm{a}_{8}, \mathrm{a}_{4}\right]\right],\left[\left[\mathrm{a}_{7}, \mathrm{a}_{3}\right],\left[\mathrm{a}_{1}, \mathrm{a}_{5}\right]\right]\right]=\left[\left[\left[\mathrm{a}_{1}, \mathrm{a}_{5}\right],\left[\mathrm{a}_{3}, \mathrm{a}_{7}\right]\right],\left[\left[\mathrm{a}_{2}, \mathrm{a}_{6}\right],\left[\mathrm{a}_{4}, \mathrm{a}_{8}\right]\right]\right]$

$\left[\left[\left[a_{7}, a_{3}\right],\left[a_{1}, a_{5}\right]\right],\left[\left[a_{8}, a_{4}\right],\left[a_{2}, a_{6}\right]\right]\right]=\left[\left[\left[a_{1}, a_{5}\right],\left[a_{3}, a_{7}\right]\right],\left[\left[a_{2}, a_{6}\right],\left[a_{4}, a_{8}\right]\right]\right]$

$\left[\left[\left[a_{8}, a_{4}\right],\left[a_{2}, a_{6}\right]\right],\left[\left[a_{1}, a_{5}\right],\left[a_{3}, a_{7}\right]\right]\right]=\left[\left[\left[a_{1}, a_{5}\right],\left[a_{3}, a_{7}\right]\right],\left[\left[a_{2}, a_{6}\right],\left[a_{4}, a_{8}\right]\right]\right]$

Step8

0 allout

Eliminating any exceptional integer according to the four inequalities in the process of recursive operations, it logically makes sense thus. However, after this paper was accepted, we detected a counter example of our conjecture to the case of $\mathrm{N}=8$ after the vast data verification. It is as follows.

$\begin{array}{lllllllll} & 1 & 2 & 3 & 4 & 5 & 6 & 7 & 8 \\ \mathrm{~S} & 0 & 0 & 0 & 0 & 1 & 1 & 3 & 3 \\ 1 & 0 & 0 & 0 & 1 & 0 & 2 & 0 & 3 \\ 2 & 0 & 0 & 1 & 1 & 2 & 2 & 3 & 3 \\ 3 & 0 & 1 & 0 & 1 & 0 & 1 & 0 & 3 \\ 4 & 1 & 1 & 1 & 1 & 1 & 1 & 3 & 3 \\ 5 & 0 & 0 & 0 & 0 & 0 & 2 & 0 & 2 \\ 6 & 0 & 0 & 0 & 0 & 2 & 2 & 2 & 2 \\ 7 & 0 & 0 & 0 & 2 & 0 & 0 & 0 & 2 \\ 8 & 0 & 0 & 2 & 2 & 0 & 0 & 2 & 2 \\ 9 & 0 & 2 & 0 & 2 & 0 & 2 & 0 & 2 \\ 10 & 2 & 2 & 2 & 2 & 2 & 2 & 2 & 2 \\ 11 & 0 & 0 & 0 & 0 & 0 & 0 & 0 & 0\end{array}$


In this case, the recursive operation takes 11 steps. According to further computational checks when $\mathrm{N}=8$ except for $\mathrm{A}=\mathrm{B}=\mathrm{C}$ at the first step, the conjecture is valid with base three numbers $(0,1,2)$ (, but it is not by congruent expression modulo 3 on the recursive operation.) On the other hand, we detects counter examples in the operations with more than base three numbers like the case mentioned above $(; 0,1,2,3$ or more.) So that, we cannot say the conjecture will be true more than the case of $\mathrm{N}=4$ at this time.

If the conjecture is discussed only with binary numbers $(0,1)$, it needs none of any four inequalities as the conditions to the conjecture. However, it is easily possible to prove such a case with binary numbers so that it will be no longer conjecture. On the other hand, the data suggests the conjecture will be valid also for the case with the three numbers $(0,1,2)$. Thus, the general conjecture still seems to make sense in such a narrow domain. At the same time, we have no idea why it does until the case with base three numbers and does not more than the case with three base numbers against the recursive operations we logically expand. Additionally to say, if consecutive integers $\mathrm{A}, \mathrm{B}, \mathrm{C}, \mathrm{D}, \mathrm{E}$, and $\mathrm{F}$ are $\mathrm{A}=\mathrm{B}<\mathrm{C}=\mathrm{D}<\mathrm{E}=\mathrm{F}$, such any sequences will not follow the conjecture: reasoning aside, Eliminating this condition from the conjecture seems to be a solution to exclude the counter example. Or, we may not notice an extra condition necessary for the conjecture. We would therefore like to know the preferable solution and offer these issues of our conjecture.

\section{References}

[1] B. Freedman, The Four Number Game, Scripta Math., 14: 35-47, (1948)

[2] M. Chamberland and D. M. Thomas, The N-Number Ducci Game(in the section Open Problems and Conjectures edited by G. Ladas), J. of Difference Equations and Applications, Vol. 10, No. 3, 339-342, (Mar. 2004). Retrieved 2009-01-26.

Supplementary Data (, N=16 in the case iii).)

$\mathrm{N}=16$,

Step 1

$\left[a_{1}, a_{2}\right],\left[a_{2}, a_{3}\right],\left[a_{3}, a_{4}\right],\left[a_{4}, a_{5}\right],\left[a_{5}, a_{6}\right],\left[a_{6}, a_{7}\right],\left[a_{7}, a_{8}\right],\left[a_{8}, a_{9}\right],\left[a_{9}, a_{10}\right],\left[a_{10}, a_{11}\right]$, $\left[\mathrm{a}_{11}, \mathrm{a}_{12}\right],\left[\mathrm{a}_{12}, \mathrm{a}_{13}\right],\left[\mathrm{a}_{13}, \mathrm{a}_{14}\right],\left[\mathrm{a}_{14}, \mathrm{a}_{15}\right],\left[\mathrm{a}_{15}, \mathrm{a}_{16}\right],\left[\mathrm{a}_{16}, \mathrm{a}_{1}\right]$

Step2

$\left[\left[a_{1}, a_{2}\right],\left[a_{2}, a_{3}\right]\right]=\left[a_{1}, a_{3}\right]$

$\left[\left[a_{2}, a_{3}\right],\left[a_{3}, a_{4}\right]\right]=\left[a_{2}, a_{4}\right]$

$\left[\left[a_{3}, a_{4}\right],\left[a_{4}, a_{5}\right]\right]=\left[a_{3}, a_{5}\right]$

$\left[\left[a_{4}, a_{5}\right],\left[a_{5}, a_{6}\right]\right]=\left[a_{4}, a_{6}\right]$

$\left[\left[a_{5}, a_{6}\right],\left[a_{6}, a_{7}\right]\right]=\left[a_{5}, a_{7}\right]$

$\left[\left[\mathrm{a}_{6}, \mathrm{a}_{7}\right],\left[\mathrm{a}_{7}, \mathrm{a}_{8}\right]\right]=\left[\mathrm{a}_{6}, \mathrm{a}_{8}\right]$

$\left[\left[a_{7}, a_{8}\right],\left[a_{8}, a_{9}\right]\right]=\left[a_{7}, a_{9}\right]$

$\left[\left[a_{8}, a_{9}\right],\left[a_{9}, a_{10}\right]\right]=\left[a_{8}, a_{10}\right]$

$\left[\left[a_{9}, a_{10}\right],\left[a_{10}, a_{11}\right]\right]=\left[a_{9}, a_{11}\right]$ 
$\left[\left[\mathrm{a}_{10}, \mathrm{a}_{11}\right],\left[\mathrm{a}_{11}, \mathrm{a}_{12}\right]\right]=\left[\mathrm{a}_{10}, \mathrm{a}_{12}\right]$

$\left[\left[a_{11}, a_{12}\right],\left[a_{12}, a_{13}\right]\right]=\left[a_{11}, a_{13}\right]$

$\left[\left[a_{12}, a_{13}\right],\left[a_{13}, a_{14}\right]\right]=\left[a_{12}, a_{14}\right]$

$\left[\left[a_{13}, a_{14}\right],\left[a_{14}, a_{15}\right]\right]=\left[a_{13}, a_{15}\right]$

$\left[\left[a_{14}, a_{15}\right],\left[a_{15}, a_{16}\right]\right]=\left[a_{14}, a_{16}\right]$

$\left[\left[a_{15}, a_{16}\right],\left[a_{16}, a_{1}\right]\right]=\left[a_{15}, a_{1}\right]$

$\left[\left[a_{16}, a_{1}\right],\left[a_{1}, a_{2}\right]\right]=\left[a_{16}, a_{2}\right]$

Step3

$\left[\left[a_{1}, a_{3}\right],\left[a_{2}, a_{4}\right]\right]$

$\left[\left[a_{2}, a_{4}\right],\left[a_{3}, a_{5}\right]\right]$

$\left[\left[a_{3}, a_{5}\right],\left[a_{4}, a_{6}\right]\right]$

$\left[\left[a_{4}, a_{6}\right],\left[a_{5}, a_{7}\right]\right]$

$\left[\left[a_{5}, a_{7}\right],\left[a_{6}, a_{8}\right]\right]$

$\left[\left[\mathrm{a}_{6}, \mathrm{a}_{8}\right],\left[\mathrm{a}_{7}, \mathrm{a}_{9}\right]\right]$

$\left[\left[a_{7}, a_{9}\right],\left[a_{8}, a_{10}\right]\right]$

$\left[\left[\mathrm{a}_{8}, \mathrm{a}_{10}\right],\left[\mathrm{a}_{9}, \mathrm{a}_{11}\right]\right]$

$\left[\left[a_{9}, a_{11}\right],\left[a_{10}, a_{12}\right]\right]$

$\left[\left[\mathrm{a}_{10}, \mathrm{a}_{12}\right],\left[\mathrm{a}_{11}, \mathrm{a}_{13}\right]\right]$

$\left[\left[a_{11}, a_{13}\right],\left[a_{12}, a_{14}\right]\right]$

$\left[\left[\mathrm{a}_{12}, \mathrm{a}_{14}\right],\left[\mathrm{a}_{13}, \mathrm{a}_{15}\right]\right]$

$\left[\left[\mathrm{a}_{13}, \mathrm{a}_{15}\right],\left[\mathrm{a}_{14}, \mathrm{a}_{16}\right]\right]$

$\left[\left[\mathrm{a}_{14}, \mathrm{a}_{16}\right],\left[\mathrm{a}_{15}, \mathrm{a}_{1}\right]\right]$

$\left[\left[\mathrm{a}_{15}, \mathrm{a}_{1}\right],\left[\mathrm{a}_{16}, \mathrm{a}_{2}\right]\right]$

$\left[\left[a_{16}, a_{2}\right],\left[a_{1}, a_{3}\right]\right]$

Step4 (Remark: any integer at vertices follows the case iii) of lemma 2.1.)

$\left[\left[\left[\mathrm{a}_{1}, \mathrm{a}_{3}\right],\left[\mathrm{a}_{2}, \mathrm{a}_{4}\right]\right],\left[\left[\mathrm{a}_{2}, \mathrm{a}_{4}\right],\left[\mathrm{a}_{3}, \mathrm{a}_{5}\right]\right]\right]=\left[\left[\mathrm{a}_{1}, \mathrm{a}_{3}\right],\left[\mathrm{a}_{3}, \mathrm{a}_{5}\right]\right]=\left[\mathrm{a}_{1}, \mathrm{a}_{5}\right]$

$\left[\left[\left[a_{2}, a_{4}\right],\left[a_{3}, a_{5}\right]\right],\left[\left[a_{3}, a_{5}\right],\left[a_{4}, a_{6}\right]\right]\right]=\left[\left[a_{2}, a_{4}\right],\left[a_{4}, a_{6}\right]\right]=\left[a_{2}, a_{6}\right]$

$\left[\left[\left[a_{3}, a_{5}\right],\left[a_{4}, a_{6}\right]\right],\left[\left[a_{4}, a_{6}\right],\left[a_{5}, a_{7}\right]\right]\right]=\left[\left[a_{3}, a_{5}\right],\left[a_{5}, a_{7}\right]\right]=\left[a_{3}, a_{7}\right]$

$\left[\left[\left[a_{4}, a_{6}\right],\left[a_{5}, a_{7}\right]\right],\left[\left[a_{5}, a_{7}\right],\left[a_{6}, a_{8}\right]\right]\right]=\left[\left[a_{4}, a_{6}\right],\left[a_{6}, a_{8}\right]\right]=\left[a_{4}, a_{8}\right]$

$\left[\left[\left[a_{5}, a_{7}\right],\left[a_{6}, a_{8}\right]\right],\left[\left[a_{6}, a_{8}\right],\left[a_{7}, a_{9}\right]\right]\right]=\left[\left[a_{5}, a_{7}\right],\left[a_{7}, a_{9}\right]\right]=\left[a_{5}, a_{9}\right]$

$\left[\left[\left[\mathrm{a}_{6}, \mathrm{a}_{8}\right],\left[\mathrm{a}_{7}, \mathrm{a}_{9}\right]\right],\left[\left[\mathrm{a}_{7}, \mathrm{a}_{9}\right],\left[\mathrm{a}_{8}, \mathrm{a}_{10}\right]\right]\right]=\left[\left[\mathrm{a}_{6}, \mathrm{a}_{8}\right],\left[\mathrm{a}_{8}, \mathrm{a}_{10}\right]\right]=\left[\mathrm{a}_{6}, \mathrm{a}_{10}\right]$

$\left[\left[\left[a_{7}, a_{9}\right],\left[a_{8}, a_{10}\right]\right],\left[\left[a_{8}, a_{10}\right],\left[a_{9}, a_{11}\right]\right]\right]=\left[\left[a_{7}, a_{9}\right],\left[a_{9}, a_{11}\right]\right]=\left[a_{7}, a_{11}\right]$

$\left[\left[\left[\mathrm{a}_{8}, \mathrm{a}_{10}\right],\left[\mathrm{a}_{9}, \mathrm{a}_{11}\right]\right],\left[\left[\mathrm{a}_{9}, \mathrm{a}_{11}\right],\left[\mathrm{a}_{10}, \mathrm{a}_{12}\right]\right]\right]=\left[\left[\mathrm{a}_{8}, \mathrm{a}_{10}\right],\left[\mathrm{a}_{10}, \mathrm{a}_{12}\right]\right]=\left[\mathrm{a}_{8}, \mathrm{a}_{12}\right]$

$\left[\left[\left[a_{9}, a_{11}\right],\left[a_{10}, a_{12}\right]\right],\left[\left[a_{10}, a_{12}\right],\left[a_{11}, a_{13}\right]\right]\right]=\left[\left[a_{9}, a_{11}\right],\left[a_{11}, a_{13}\right]\right]=\left[a_{9}, a_{13}\right]$

$\left[\left[\left[\mathrm{a}_{10}, \mathrm{a}_{12}\right],\left[\mathrm{a}_{11}, \mathrm{a}_{13}\right]\right],\left[\left[\mathrm{a}_{11}, \mathrm{a}_{13}\right],\left[\mathrm{a}_{12}, \mathrm{a}_{14}\right]\right]\right]=\left[\left[\mathrm{a}_{10}, \mathrm{a}_{12}\right],\left[\mathrm{a}_{12}, \mathrm{a}_{14}\right]\right]=\left[\mathrm{a}_{10}, \mathrm{a}_{14}\right]$

$\left[\left[\left[\mathrm{a}_{11}, \mathrm{a}_{13}\right],\left[\mathrm{a}_{12}, \mathrm{a}_{14}\right]\right],\left[\left[\mathrm{a}_{12}, \mathrm{a}_{14}\right],\left[\mathrm{a}_{13}, \mathrm{a}_{15}\right]\right]\right]=\left[\left[\mathrm{a}_{11}, \mathrm{a}_{13}\right],\left[\mathrm{a}_{13}, \mathrm{a}_{15}\right]\right]=\left[\mathrm{a}_{11}, \mathrm{a}_{15}\right]$

$\left[\left[\left[a_{12}, a_{14}\right],\left[a_{13}, a_{15}\right]\right],\left[\left[a_{13}, a_{15}\right],\left[a_{14}, a_{16}\right]\right]\right]=\left[\left[a_{12}, a_{14}\right],\left[a_{14}, a_{16}\right]\right]=\left[a_{12}, a_{16}\right]$

$\left[\left[\left[\mathrm{a}_{13}, \mathrm{a}_{15}\right],\left[\mathrm{a}_{14}, \mathrm{a}_{16}\right],\left[\left[\mathrm{a}_{14}, \mathrm{a}_{16}\right],\left[\mathrm{a}_{15}, \mathrm{a}_{1}\right]\right]\right]=\left[\left[\mathrm{a}_{13}, \mathrm{a}_{15}\right],\left[\mathrm{a}_{15}, \mathrm{a}_{1}\right]\right]=\left[\mathrm{a}_{13}, \mathrm{a}_{1}\right]\right.$

$\left[\left[\left[a_{14}, a_{16}\right],\left[a_{15}, a_{1}\right]\right],\left[\left[a_{15}, a_{1}\right],\left[a_{16}, a_{2}\right]\right]\right]=\left[\left[a_{14}, a_{16}\right],\left[a_{16}, a_{2}\right]\right]=\left[a_{14}, a_{2}\right]$

$\left[\left[\left[\mathrm{a}_{15}, \mathrm{a}_{1}\right],\left[\mathrm{a}_{16}, \mathrm{a}_{2}\right]\right],\left[\left[\mathrm{a}_{16}, \mathrm{a}_{2}\right],\left[\mathrm{a}_{1}, \mathrm{a}_{3}\right]\right]\right]=\left[\left[\mathrm{a}_{15}, \mathrm{a}_{1}\right],\left[\mathrm{a}_{1}, \mathrm{a}_{3}\right]\right]=\left[\mathrm{a}_{15}, \mathrm{a}_{3}\right]$

$\left[\left[\left[a_{16}, a_{2}\right],\left[a_{1}, a_{3}\right]\right],\left[\left[a_{1}, a_{3}\right],\left[a_{2}, a_{4}\right]\right]\right]=\left[\left[a_{16}, a_{2}\right],\left[a_{2}, a_{4}\right]\right]=\left[a_{16}, a_{4}\right]$

Step5

$\left[\left[a_{1}, a_{5}\right],\left[a_{2}, a_{6}\right]\right]$

$\left[\left[a_{2}, a_{6}\right],\left[a_{3}, a_{7}\right]\right]$

$\left[\left[a_{3}, a_{7}\right],\left[a_{4}, a_{8}\right]\right]$ 
$\left[\left[a_{4}, a_{8}\right],\left[a_{5}, a_{9}\right]\right]$
$\left[\left[a_{5}, a_{1}\right],\left[a_{6}, a_{10}\right]\right]$
$\left[\left[a_{6}, a_{2}\right],\left[a_{7}, a_{11}\right]\right]$
$\left[\left[a_{7}, a_{3}\right],\left[a_{8}, a_{12}\right]\right]$
$\left[\left[a_{8}, a_{4}\right],\left[a_{9}, a_{13}\right]\right]$
$\left[\left[a_{9}, a_{5}\right],\left[a_{10}, a_{14}\right]\right]$
$\left[\left[a_{10}, a_{6}\right],\left[a_{11}, a_{15}\right]\right]$
$\left[\left[a_{11}, a_{7}\right],\left[a_{12}, a_{16}\right]\right]$
$\left[\left[a_{12}, a_{8}\right],\left[a_{13}, a_{1}\right]\right]$
$\left[\left[a_{13}, a_{1}\right],\left[a_{14}, a_{2}\right]\right]$
$\left[\left[a_{14}, a_{2}\right],\left[a_{15}, a_{3}\right]\right]$
$\left[\left[a_{15}, a_{3}\right],\left[a_{16}, a_{4}\right]\right]$
$\left[\left[a_{16}, a_{4}\right],\left[a_{1}, a_{5}\right]\right]$

Step6 (Remark: any integer at vertices follows the case iii) of lemma 2.1.)

$\left[\left[\left[a_{1}, a_{5}\right],\left[a_{2}, a_{6}\right]\right],\left[\left[a_{2}, a_{6}\right],\left[a_{3}, a_{7}\right]\right]\right]=\left[\left[a_{1}, a_{5}\right],\left[a_{3}, a_{7}\right]\right]$

$\left[\left[\left[a_{2}, a_{6}\right],\left[a_{3}, a_{7}\right]\right],\left[\left[a_{3}, a_{7}\right],\left[a_{4}, a_{8}\right]\right]\right]=\left[\left[a_{2}, a_{6}\right],\left[a_{4}, a_{8}\right]\right]$

$\left[\left[\left[a_{3}, a_{7}\right],\left[a_{4}, a_{8}\right]\right],\left[\left[a_{4}, a_{8}\right],\left[a_{5}, a_{9}\right]\right]\right]=\left[\left[a_{3}, a_{7}\right],\left[a_{5}, a_{9}\right]\right]$

$\left[\left[\left[\mathrm{a}_{4}, \mathrm{a}_{8}\right],\left[\mathrm{a}_{5}, \mathrm{a}_{9}\right]\right],\left[\left[\mathrm{a}_{5}, \mathrm{a}_{9}\right],\left[\mathrm{a}_{6}, \mathrm{a}_{10}\right]\right]\right]=\left[\left[\mathrm{a}_{4}, \mathrm{a}_{8}\right],\left[\mathrm{a}_{6}, \mathrm{a}_{10}\right]\right]$

$\left[\left[\left[a_{5}, a_{9}\right],\left[a_{6}, a_{10}\right]\right],\left[\left[a_{6}, a_{10}\right],\left[a_{7}, a_{11}\right]\right]\right]=\left[\left[a_{5}, a_{9}\right],\left[a_{7}, a_{11}\right]\right]$

$\left[\left[\left[\mathrm{a}_{6}, \mathrm{a}_{10}\right],\left[\mathrm{a}_{7}, \mathrm{a}_{11}\right]\right],\left[\left[\mathrm{a}_{7}, \mathrm{a}_{11}\right],\left[\mathrm{a}_{8}, \mathrm{a}_{12}\right]\right]\right]=\left[\left[\mathrm{a}_{6}, \mathrm{a}_{10}\right],\left[\mathrm{a}_{8}, \mathrm{a}_{12}\right]\right]$

$\left[\left[\left[\mathrm{a}_{7}, \mathrm{a}_{11}\right],\left[\mathrm{a}_{8}, \mathrm{a}_{12}\right]\right],\left[\left[\mathrm{a}_{8}, \mathrm{a}_{12}\right],\left[\mathrm{a}_{9}, \mathrm{a}_{13}\right]\right]\right]=\left[\left[\mathrm{a}_{7}, \mathrm{a}_{11}\right],\left[\mathrm{a}_{9}, \mathrm{a}_{13}\right]\right]$

$\left[\left[\left[a_{8}, a_{12}\right],\left[a_{9}, a_{13}\right]\right],\left[\left[a_{9}, a_{13}\right],\left[a_{10}, a_{14}\right]\right]\right]=\left[\left[a_{8}, a_{12}\right],\left[a_{10}, a_{14}\right]\right]$

$\left[\left[\left[a_{1}, a_{13}\right],\left[a_{10}, a_{14}\right]\right],\left[\left[a_{10}, a_{14}\right],\left[a_{11}, a_{15}\right]\right]\right]=\left[\left[a_{9}, a_{13}\right],\left[a_{11}, a_{15}\right]\right]$

$\left[\left[\left[a_{2}, a_{14}\right],\left[a_{11}, a_{15}\right]\right],\left[\left[a_{11}, a_{15}\right],\left[a_{12}, a_{16}\right]\right]\right]=\left[\left[a_{10}, a_{14}\right],\left[a_{12}, a_{16}\right]\right]$

$\left[\left[\left[\mathrm{a}_{3}, \mathrm{a}_{15}\right],\left[\mathrm{a}_{12}, \mathrm{a}_{16}\right]\right],\left[\left[\mathrm{a}_{12}, \mathrm{a}_{16}\right],\left[\mathrm{a}_{13}, \mathrm{a}_{1}\right]\right]\right]=\left[\left[\mathrm{a}_{11}, \mathrm{a}_{15}\right],\left[\mathrm{a}_{13}, \mathrm{a}_{1}\right]\right]$

$\left[\left[\left[\mathrm{a}_{4}, \mathrm{a}_{16}\right],\left[\mathrm{a}_{13}, \mathrm{a}_{1}\right]\right],\left[\left[\mathrm{a}_{13}, \mathrm{a}_{1}\right],\left[\mathrm{a}_{14}, \mathrm{a}_{2}\right]\right]\right]=\left[\left[\mathrm{a}_{12}, \mathrm{a}_{16}\right],\left[\mathrm{a}_{14}, \mathrm{a}_{2}\right]\right]$

$\left[\left[\left[a_{5}, a_{1}\right],\left[a_{14}, a_{2}\right]\right],\left[\left[a_{14}, a_{2}\right],\left[a_{15}, a_{3}\right]\right]\right]=\left[\left[a_{13}, a_{1}\right],\left[a_{15}, a_{3}\right]\right]$

$\left[\left[\left[a_{6}, a_{2}\right],\left[a_{15}, a_{3}\right]\right],\left[\left[a_{15}, a_{3}\right],\left[a_{16}, a_{4}\right]\right]\right]=\left[\left[a_{14}, a_{2}\right],\left[a_{16}, a_{4}\right]\right]$

$\left[\left[\left[a_{7}, a_{3}\right],\left[a_{16}, a_{4}\right]\right],\left[\left[a_{16}, a_{4}\right],\left[a_{1}, a_{5}\right]\right]\right]=\left[\left[a_{15}, a_{3}\right],\left[a_{1}, a_{5}\right]\right]$

$\left[\left[\left[a_{8}, a_{4}\right],\left[a_{1}, a_{5}\right]\right],\left[\left[a_{1}, a_{5}\right],\left[a_{2}, a_{6}\right]\right]\right]=\left[\left[a_{16}, a_{4}\right],\left[a_{2}, a_{6}\right]\right]$

Step7

$\left[\left[\left[a_{1}, a_{5}\right],\left[a_{3}, a_{7}\right]\right],\left[\left[a_{2}, a_{6}\right],\left[a_{4}, a_{8}\right]\right]\right]$

$\left[\left[\left[a_{2}, a_{6}\right],\left[a_{4}, a_{8}\right]\right],\left[\left[a_{3}, a_{7}\right],\left[a_{5}, a_{9}\right]\right]\right]$

$\left[\left[\left[a_{3}, a_{7}\right],\left[a_{5}, a_{9}\right]\right],\left[\left[a_{4}, a_{8}\right],\left[a_{6}, a_{10}\right]\right]\right]$

$\left[\left[\left[\mathrm{a}_{4}, \mathrm{a}_{8}\right],\left[\mathrm{a}_{6}, \mathrm{a}_{10}\right]\right],\left[\left[\mathrm{a}_{5}, \mathrm{a}_{9}\right],\left[\mathrm{a}_{7}, \mathrm{a}_{11}\right]\right]\right]$

$\left[\left[\left[a_{5}, a_{9}\right],\left[a_{7}, a_{11}\right]\right],\left[\left[a_{6}, a_{10}\right],\left[a_{8}, a_{12}\right]\right]\right]$

$\left[\left[\left[a_{6}, a_{10}\right],\left[a_{8}, a_{12}\right]\right],\left[\left[a_{7}, a_{11}\right],\left[a_{9}, a_{13}\right]\right]\right]$

$\left[\left[\left[a_{7}, a_{11}\right],\left[a_{9}, a_{13}\right]\right],\left[\left[a_{8}, a_{12}\right],\left[a_{10}, a_{14}\right]\right]\right]$

$\left[\left[\left[a_{8}, a_{12}\right],\left[a_{10}, a_{14}\right]\right],\left[\left[a_{9}, a_{13}\right],\left[a_{11}, a_{15}\right]\right]\right]$

$\left[\left[\left[\mathrm{a}_{9}, \mathrm{a}_{13}\right],\left[\mathrm{a}_{11}, \mathrm{a}_{15}\right]\right],\left[\left[\mathrm{a}_{10}, \mathrm{a}_{14}\right],\left[\mathrm{a}_{12}, \mathrm{a}_{16}\right]\right]\right]$

$\left[\left[\left[\mathrm{a}_{10}, \mathrm{a}_{14}\right],\left[\mathrm{a}_{12}, \mathrm{a}_{15}\right]\right],\left[\left[\mathrm{a}_{11}, \mathrm{a}_{15}\right],\left[\mathrm{a}_{13}, \mathrm{a}_{1}\right]\right]\right]$

$\left[\left[\left[a_{11}, a_{15}\right],\left[a_{13}, a_{1}\right]\right],\left[\left[a_{12}, a_{16}\right],\left[a_{14}, a_{2}\right]\right]\right]$

$\left[\left[\left[a_{12}, a_{16}\right],\left[a_{14}, a_{2}\right]\right],\left[\left[a_{13}, a_{1}\right],\left[a_{15}, a_{3}\right]\right]\right]$

$\left[\left[\left[a_{13}, a_{1}\right],\left[a_{15}, a_{3}\right]\right],\left[\left[a_{14}, a_{2}\right],\left[a_{15}, a_{4}\right]\right]\right]$

$\left[\left[\left[a_{14}, a_{2}\right],\left[a_{16}, a_{4}\right]\right],\left[\left[a_{15}, a_{3}\right],\left[a_{1}, a_{5}\right]\right]\right]$ 
$\left[\left[\left[a_{15}, a_{3}\right],\left[a_{1}, a_{5}\right]\right],\left[\left[a_{16}, a_{4}\right],\left[a_{2}, a_{6}\right]\right]\right]$

$\left[\left[\left[\mathrm{a}_{16}, \mathrm{a}_{4}\right],\left[\mathrm{a}_{2}, \mathrm{a}_{6}\right]\right],\left[\left[\mathrm{a}_{1}, \mathrm{a}_{5}\right],\left[\mathrm{a}_{3}, \mathrm{a}_{7}\right]\right]\right]$

Step8 (Remark: any integer at vertices follows the case iii) of lemma 2.1.)

$\left[\left[\left[\left[a_{1}, a_{5}\right],\left[a_{3}, a_{7}\right]\right],\left[\left[a_{2}, a_{6}\right],\left[a_{4}, a_{8}\right]\right]\right],\left[\left[\left[a_{2}, a_{6}\right],\left[a_{4}, a_{8}\right]\right],\left[\left[a_{3}, a_{7}\right],\left[a_{5}, a_{9}\right]\right]\right]\right]=\left[a_{1}, a_{9}\right]$

$\left[\left[\left[\left[a_{2}, a_{6}\right],\left[a_{4}, a_{8}\right]\right],\left[\left[a_{3}, a_{7}\right],\left[a_{5}, a_{9}\right]\right]\right],\left[\left[\left[a_{3}, a_{7}\right],\left[a_{5}, a_{9}\right]\right],\left[\left[a_{4}, a_{8}\right],\left[a_{6}, a_{10}\right]\right]\right]\right]=\left[a_{2}, a_{10}\right]$

$\left[\left[\left[\left[a_{3}, a_{7}\right],\left[a_{5}, a_{9}\right]\right],\left[\left[a_{4}, a_{8}\right],\left[a_{6}, a_{10}\right]\right]\right],\left[\left[\left[a_{4}, a_{8}\right],\left[a_{6}, a_{10}\right]\right],\left[\left[a_{5}, a_{9}\right],\left[a_{7}, a_{11}\right]\right]\right]=\left[a_{3}, a_{11}\right]\right.$

$\left[\left[\left[\left[a_{4}, a_{8}\right],\left[a_{6}, a_{10}\right]\right],\left[\left[a_{5}, a_{9}\right],\left[a_{7}, a_{11}\right]\right]\right],\left[\left[\left[a_{5}, a_{9}\right],\left[a_{7}, a_{11}\right]\right],\left[\left[a_{6}, a_{10}\right],\left[a_{8}, a_{12}\right]\right]\right]=\left[a_{4}\right.\right.$, $\left.\mathrm{a}_{12}\right]$

$\left[\left[\left[\left[a_{5}, a_{9}\right],\left[a_{7}, a_{11}\right]\right],\left[\left[a_{6}, a_{10}\right],\left[a_{8}, a_{12}\right]\right]\right],\left[\left[\left[a_{6}, a_{10}\right],\left[a_{8}, a_{12}\right]\right],\left[\left[a_{7}, a_{11}\right],\left[a_{9}, a_{13}\right]\right]\right]\right]=\left[a_{5}\right.$, $\left.\mathrm{a}_{13}\right]$

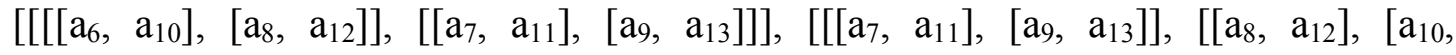
$\left.\left.\left.\left.\mathrm{a}_{14}\right]\right]\right]\right]=\left[\mathrm{a}_{6}, \mathrm{a}_{14}\right]$

$\left[\left[\left[\left[a_{7}, a_{11}\right],\left[a_{9}, a_{13}\right]\right],\left[\left[a_{8}, a_{12}\right],\left[a_{10}, a_{14}\right]\right]\right],\left[\left[\left[a_{8}, a_{12}\right],\left[a_{10}, a_{14}\right]\right],\left[\left[a_{9}, a_{13}\right],\left[a_{11}\right.\right.\right.\right.$, $\left.\left.\left.\left.\mathrm{a}_{15}\right]\right]\right]\right]=\left[\mathrm{a}_{7}, \mathrm{a}_{15}\right]$

$\left[\left[\left[\left[a_{8}, a_{12}\right],\left[a_{10}, a_{14}\right]\right],\left[\left[a_{9}, a_{13}\right],\left[a_{11}, a_{15}\right]\right]\right],\left[\left[\left[a_{9}, a_{13}\right],\left[a_{11}, a_{15}\right]\right],\left[\left[a_{10}, a_{14}\right],\left[a_{12}\right.\right.\right.\right.$, $\left.\left.\left.\left.\mathrm{a}_{16}\right]\right]\right]\right]=\left[\mathrm{a}_{8}, \mathrm{a}_{16}\right]$

$\left[\left[\left[\left[a_{9}, a_{13}\right],\left[a_{11}, a_{15}\right]\right],\left[\left[a_{10}, a_{14}\right],\left[a_{12}, a_{16}\right]\right]\right],\left[\left[\left[a_{10}, a_{14}\right],\left[a_{12}, a_{15}\right]\right],\left[\left[a_{11}, a_{15}\right],\left[a_{13}\right.\right.\right.\right.$, $\left.\left.\left.\left.a_{1}\right]\right]\right]\right]=\left[a_{9}, a_{1}\right]$

$\left[\left[\left[\left[a_{10}, a_{14}\right],\left[a_{12}, a_{15}\right]\right],\left[\left[a_{11}, a_{15}\right],\left[a_{13}, a_{1}\right]\right]\right],\left[\left[\left[a_{11}, a_{15}\right],\left[a_{13}, a_{1}\right]\right],\left[\left[a_{12}, a_{16}\right],\left[a_{14}\right.\right.\right.\right.$, $\left.\left.\left.\left.\mathrm{a}_{2}\right]\right]\right]\right]=\left[\mathrm{a}_{10}, \mathrm{a}_{2}\right]$

$\left[\left[\left[\left[a_{11}, a_{15}\right],\left[a_{13}, a_{1}\right]\right],\left[\left[a_{12}, a_{16}\right],\left[a_{14}, a_{2}\right]\right]\right],\left[\left[\left[a_{12}, a_{16}\right],\left[a_{14}, a_{2}\right]\right],\left[\left[a_{13}, a_{1}\right],\left[a_{15}\right.\right.\right.\right.$, $\left.\left.\left.\left.\mathrm{a}_{3}\right]\right]\right]\right]=\left[\mathrm{a}_{11}, \mathrm{a}_{3}\right]$

$\left[\left[\left[\left[a_{12}, a_{16}\right],\left[a_{14}, a_{2}\right]\right],\left[\left[a_{13}, a_{1}\right],\left[a_{15}, a_{3}\right]\right]\right],\left[\left[\left[a_{13}, a_{1}\right],\left[a_{15}, a_{3}\right]\right],\left[\left[a_{14}, a_{2}\right],\left[a_{15}\right.\right.\right.\right.$, $\left.\left.\left.\left.a_{4}\right]\right]\right]\right]=\left[a_{12}, a_{4}\right]$

$\left.\left[\left[\left[a_{13}, a_{1}\right],\left[a_{15}, a_{3}\right]\right],\left[\left[a_{14}, a_{2}\right],\left[a_{15}, a_{4}\right]\right]\right],\left[\left[\left[a_{14}, a_{2}\right],\left[a_{16}, a_{4}\right]\right],\left[\left[a_{15}, a_{3}\right],\left[a_{1}, a_{5}\right]\right]\right]\right]=\left[a_{13}\right.$, $\left.a_{5}\right]$

$\left[\left[\left[\left[a_{14}, a_{2}\right],\left[a_{16}, a_{4}\right]\right],\left[\left[a_{15}, a_{3}\right],\left[a_{1}, a_{5}\right]\right]\right],\left[\left[\left[a_{15}, a_{3}\right],\left[a_{1}, a_{5}\right]\right],\left[\left[a_{16}, a_{4}\right],\left[a_{2}, a_{6}\right]\right]\right]\right]=\left[a_{14}\right.$, $\left.\mathrm{a}_{6}\right]$

[[[[a $\left.\left.\left.\left.a_{15}, a_{3}\right],\left[a_{1}, a_{5}\right]\right],\left[\left[a_{16}, a_{4}\right],\left[a_{2}, a_{6}\right]\right]\right],\left[\left[\left[a_{16}, a_{4}\right],\left[a_{2}, a_{6}\right]\right],\left[\left[a_{1}, a_{5}\right],\left[a_{3}, a_{7}\right]\right]\right]\right]=\left[a_{15}, a_{7}\right]$ $\left[\left[\left[\left[a_{16}, a_{4}\right],\left[a_{2}, a_{6}\right]\right],\left[\left[a_{1}, a_{5}\right],\left[a_{3}, a_{7}\right]\right]\right],\left[\left[\left[a_{1}, a_{5}\right],\left[a_{3}, a_{7}\right]\right],\left[\left[a_{2}, a_{6}\right],\left[a_{4}, a_{8}\right]\right]\right]\right]=\left[a_{16}, a_{8}\right]$

Step9

$\left[\left[\mathrm{a}_{1}, \mathrm{a}_{9}\right],\left[\mathrm{a}_{2}, \mathrm{a}_{10}\right]\right]$

$\left[\left[\mathrm{a}_{2}, \mathrm{a}_{10}\right],\left[\mathrm{a}_{3}, \mathrm{a}_{11}\right]\right]$

$\left[\left[\mathrm{a}_{3}, \mathrm{a}_{11}\right],\left[\mathrm{a}_{4}, \mathrm{a}_{12}\right]\right]$

$\left[\left[\mathrm{a}_{4}, \mathrm{a}_{12}\right],\left[\mathrm{a}_{5}, \mathrm{a}_{13}\right]\right]$

$\left[\left[\mathrm{a}_{5}, \mathrm{a}_{13}\right],\left[\mathrm{a}_{6}, \mathrm{a}_{14}\right]\right]$

$\left[\left[\mathrm{a}_{6}, \mathrm{a}_{14}\right],\left[\mathrm{a}_{7}, \mathrm{a}_{15}\right]\right]$

$\left[\left[\mathrm{a}_{7}, \mathrm{a}_{15}\right],\left[\mathrm{a}_{8}, \mathrm{a}_{16}\right]\right]$

$\left[\left[a_{8}, a_{16}\right],\left[a_{9}, a_{1}\right]\right]$

$\left[\left[a_{9}, a_{1}\right],\left[a_{10}, a_{2}\right]\right]$

$\left[\left[a_{10}, a_{2}\right],\left[a_{11}, a_{3}\right]\right]$

$\left[\left[\mathrm{a}_{11}, \mathrm{a}_{3}\right],\left[\mathrm{a}_{12}, \mathrm{a}_{4}\right]\right]$

$\left[\left[a_{12}, a_{4}\right],\left[a_{13}, a_{5}\right]\right]$

$\left[\left[a_{13}, a_{5}\right],\left[a_{14}, a_{6}\right]\right]$

$\left[\left[a_{14}, a_{6}\right],\left[a_{15}, a_{7}\right]\right]$ 
$\left[\left[\mathrm{a}_{15}, \mathrm{a}_{7}\right],\left[\mathrm{a}_{16}, \mathrm{a}_{8}\right]\right]$

$\left[\left[\mathrm{a}_{16}, \mathrm{a}_{8}\right],\left[\mathrm{a}_{1}, \mathrm{a}_{9}\right]\right]$

Step10 (Remark: any integer at vertices follows the case iii) of lemma 2.1.)

$\left[\left[\left[\mathrm{a}_{1}, \mathrm{a}_{9}\right],\left[\mathrm{a}_{2}, \mathrm{a}_{10}\right]\right],\left[\left[\mathrm{a}_{2}, \mathrm{a}_{10}\right],\left[\mathrm{a}_{3}, \mathrm{a}_{11}\right]\right]\right]=\left[\left[\mathrm{a}_{1}, \mathrm{a}_{9}\right],\left[\mathrm{a}_{3}, \mathrm{a}_{11}\right]\right]$

$\left[\left[\left[\mathrm{a}_{2}, \mathrm{a}_{10}\right],\left[\mathrm{a}_{3}, \mathrm{a}_{11}\right]\right],\left[\left[\mathrm{a}_{3}, \mathrm{a}_{11}\right],\left[\mathrm{a}_{4}, \mathrm{a}_{12}\right]\right]\right]=\left[\left[\mathrm{a}_{2}, \mathrm{a}_{10}\right],\left[\mathrm{a}_{4}, \mathrm{a}_{12}\right]\right]$

$\left[\left[\left[a_{3}, a_{11}\right],\left[a_{4}, a_{12}\right]\right],\left[\left[a_{4}, a_{12}\right],\left[a_{5}, a_{13}\right]\right]\right]=\left[\left[a_{3}, a_{11}\right],\left[a_{5}, a_{13}\right]\right]$

$\left[\left[\left[\mathrm{a}_{4}, \mathrm{a}_{12}\right],\left[\mathrm{a}_{5}, \mathrm{a}_{13}\right]\right],\left[\left[\mathrm{a}_{5}, \mathrm{a}_{13}\right],\left[\mathrm{a}_{6}, \mathrm{a}_{14}\right]\right]\right]=\left[\left[\mathrm{a}_{4}, \mathrm{a}_{12}\right],\left[\mathrm{a}_{6}, \mathrm{a}_{14}\right]\right]$

$\left[\left[\left[\mathrm{a}_{5}, \mathrm{a}_{13}\right],\left[\mathrm{a}_{6}, \mathrm{a}_{14}\right]\right],\left[\left[\mathrm{a}_{6}, \mathrm{a}_{14}\right],\left[\mathrm{a}_{7}, \mathrm{a}_{15}\right]\right]\right]=\left[\left[\mathrm{a}_{5}, \mathrm{a}_{13}\right],\left[\mathrm{a}_{7}, \mathrm{a}_{15}\right]\right]$

$\left[\left[\left[\mathrm{a}_{6}, \mathrm{a}_{14}\right],\left[\mathrm{a}_{7}, \mathrm{a}_{15}\right]\right],\left[\left[\mathrm{a}_{7}, \mathrm{a}_{15}\right],\left[\mathrm{a}_{8}, \mathrm{a}_{16}\right]\right]\right]=\left[\left[\mathrm{a}_{6}, \mathrm{a}_{14}\right],\left[\mathrm{a}_{8}, \mathrm{a}_{16}\right]\right]$

$\left[\left[\left[a_{7}, a_{15}\right],\left[a_{8}, a_{16}\right]\right],\left[\left[a_{8}, a_{16}\right],\left[a_{9}, a_{1}\right]\right]\right]=\left[\left[a_{7}, a_{15}\right],\left[a_{9}, a_{1}\right]\right]$

$\left[\left[\left[a_{8}, a_{16}\right],\left[a_{9}, a_{1}\right]\right],\left[\left[a_{9}, a_{1}\right],\left[a_{10}, a_{2}\right]\right]\right]=\left[\left[a_{8}, a_{16}\right],\left[a_{10}, a_{2}\right]\right]$

$\left[\left[\left[a_{9}, a_{1}\right],\left[a_{10}, a_{2}\right]\right],\left[\left[a_{10}, a_{2}\right],\left[a_{11}, a_{3}\right]\right]\right]=\left[\left[a_{9}, a_{1}\right],\left[a_{11}, a_{3}\right]\right]$

$\left[\left[\left[a_{10}, a_{2}\right],\left[a_{11}, a_{3}\right]\right],\left[\left[a_{11}, a_{3}\right],\left[a_{12}, a_{4}\right]\right]\right]=\left[\left[a_{10}, a_{2}\right],\left[a_{12}, a_{4}\right]\right]$

$\left[\left[\left[a_{11}, a_{3}\right],\left[a_{12}, a_{4}\right]\right],\left[\left[a_{12}, a_{4}\right],\left[a_{13}, a_{5}\right]\right]\right]=\left[\left[a_{11}, a_{3}\right],\left[a_{13}, a_{5}\right]\right]$

$\left[\left[\left[a_{12}, a_{4}\right],\left[a_{13}, a_{5}\right]\right],\left[\left[a_{13}, a_{5}\right],\left[a_{14}, a_{6}\right]\right]\right]=\left[\left[a_{12}, a_{4}\right],\left[a_{14}, a_{6}\right]\right]$

$\left[\left[\left[\mathrm{a}_{13}, \mathrm{a}_{5}\right],\left[\mathrm{a}_{14}, \mathrm{a}_{6}\right]\right],\left[\left[\mathrm{a}_{14}, \mathrm{a}_{6}\right],\left[\mathrm{a}_{15}, \mathrm{a}_{7}\right]\right]\right]=\left[\left[\mathrm{a}_{13}, \mathrm{a}_{5}\right],\left[\mathrm{a}_{15}, \mathrm{a}_{7}\right]\right]$

$\left[\left[\left[\mathrm{a}_{14}, \mathrm{a}_{6}\right],\left[\mathrm{a}_{15}, \mathrm{a}_{7}\right]\right],\left[\left[\mathrm{a}_{15}, \mathrm{a}_{7}\right],\left[\mathrm{a}_{16}, \mathrm{a}_{8}\right]\right]\right]=\left[\left[\mathrm{a}_{14}, \mathrm{a}_{6}\right],\left[\mathrm{a}_{16}, \mathrm{a}_{8}\right]\right]$

$\left[\left[\left[\mathrm{a}_{15}, \mathrm{a}_{7}\right],\left[\mathrm{a}_{16}, \mathrm{a}_{8}\right]\right],\left[\left[\mathrm{a}_{16}, \mathrm{a}_{8}\right],\left[\mathrm{a}_{1}, \mathrm{a}_{9}\right]\right]\right]=\left[\left[\mathrm{a}_{15}, \mathrm{a}_{7}\right],\left[\mathrm{a}_{1}, \mathrm{a}_{9}\right]\right]$

$\left[\left[\left[a_{16}, a_{8}\right],\left[a_{1}, a_{9}\right]\right],\left[\left[a_{1}, a_{9}\right],\left[a_{2}, a_{10}\right]\right]\right]=\left[\left[a_{16}, a_{8}\right],\left[a_{2}, a_{10}\right]\right]$

Step 11

$\left[\left[\left[a_{1}, a_{9}\right],\left[a_{3}, a_{11}\right]\right],\left[\left[a_{2}, a_{10}\right],\left[a_{4}, a_{12}\right]\right]\right]$

$\left[\left[\left[\mathrm{a}_{2}, \mathrm{a}_{10}\right],\left[\mathrm{a}_{4}, \mathrm{a}_{12}\right]\right],\left[\left[\mathrm{a}_{3}, \mathrm{a}_{11}\right],\left[\mathrm{a}_{5}, \mathrm{a}_{13}\right]\right]\right]$

$\left[\left[\left[a_{3}, a_{11}\right],\left[a_{5}, a_{13}\right]\right],\left[\left[a_{4}, a_{12}\right],\left[a_{6}, a_{14}\right]\right]\right]$

$\left[\left[\left[a_{4}, a_{12}\right],\left[a_{6}, a_{14}\right]\right],\left[\left[a_{5}, a_{13}\right],\left[a_{7}, a_{15}\right]\right]\right]$

$\left[\left[\left[a_{5}, a_{13}\right],\left[a_{7}, a_{15}\right]\right],\left[\left[a_{6}, a_{14}\right],\left[a_{8}, a_{16}\right]\right]\right]$

$\left[\left[\left[a_{6}, a_{14}\right],\left[a_{8}, a_{16}\right]\right],\left[\left[a_{7}, a_{15}\right],\left[a_{9}, a_{1}\right]\right]\right]$

$\left[\left[\left[a_{7}, a_{15}\right],\left[a_{9}, a_{1}\right]\right],\left[\left[a_{8}, a_{16}\right],\left[a_{10}, a_{2}\right]\right]\right]$

$\left[\left[\left[a_{8}, a_{16}\right],\left[a_{10}, a_{2}\right]\right],\left[\left[a_{9}, a_{1}\right],\left[a_{11}, a_{3}\right]\right]\right]$

$\left[\left[\left[a_{9}, a_{1}\right],\left[a_{11}, a_{3}\right]\right],\left[\left[a_{10}, a_{2}\right],\left[a_{12}, a_{4}\right]\right]\right]$

$\left[\left[\left[a_{10}, a_{2}\right],\left[a_{12}, a_{4}\right]\right],\left[\left[a_{11}, a_{3}\right],\left[a_{13}, a_{5}\right]\right]\right]$

$\left[\left[\left[a_{11}, a_{3}\right],\left[a_{13}, a_{5}\right]\right],\left[\left[a_{12}, a_{4}\right],\left[a_{14}, a_{6}\right]\right]\right]$

$\left[\left[\left[a_{12}, a_{4}\right],\left[a_{14}, a_{6}\right]\right],\left[\left[a_{13}, a_{5}\right],\left[a_{15}, a_{7}\right]\right]\right]$

$\left[\left[\left[a_{13}, a_{5}\right],\left[a_{15}, a_{7}\right]\right],\left[\left[a_{14}, a_{6}\right],\left[a_{16}, a_{8}\right]\right]\right]$

$\left[\left[\left[a_{14}, a_{6}\right],\left[a_{16}, a_{8}\right]\right],\left[\left[a_{15}, a_{7}\right],\left[a_{1}, a_{9}\right]\right]\right]$

$\left[\left[\left[a_{15}, a_{7}\right],\left[a_{1}, a_{9}\right]\right],\left[\left[a_{16}, a_{8}\right],\left[a_{2}, a_{10}\right]\right]\right]$

$\left[\left[\left[a_{16}, a_{8}\right],\left[a_{2}, a_{10}\right]\right],\left[\left[a_{1}, a_{9}\right],\left[a_{3}, a_{11}\right]\right]\right]$

Step12 (Remark: any integer at vertices follows the case iii) of lemma 2.1.)

$\left[\left[\left[\left[a_{1}, a_{9}\right],\left[a_{3}, a_{11}\right]\right],\left[\left[a_{2}, a_{10}\right],\left[a_{4}, a_{12}\right]\right]\right],\left[\left[\left[a_{2}, a_{10}\right],\left[a_{4}, a_{12}\right]\right],\left[\left[a_{3}, a_{11}\right],\left[a_{5}, a_{13}\right]\right]\right]\right]=\left[\left[a_{1}\right.\right.$, $\left.\left.\mathrm{a}_{9}\right],\left[\mathrm{a}_{5}, \mathrm{a}_{13}\right]\right]$

$\left[\left[\left[\left[a_{2}, a_{10}\right],\left[a_{4}, a_{12}\right]\right],\left[\left[a_{3}, a_{11}\right],\left[a_{5}, a_{13}\right]\right]\right],\left[\left[\left[a_{3}, a_{11}\right],\left[a_{5}, a_{13}\right]\right],\left[\left[a_{4}, a_{12}\right],\left[a_{6}\right.\right.\right.\right.$, $\left.\left.\left.\left.\mathrm{a}_{14}\right]\right]\right]\right]=\left[\left[\mathrm{a}_{2}, \mathrm{a}_{10}\right],\left[\mathrm{a}_{6}, \mathrm{a}_{14}\right]\right]$

$\left[\left[\left[\left[a_{3}, a_{11}\right],\left[a_{5}, a_{13}\right]\right],\left[\left[a_{4}, a_{12}\right],\left[a_{6}, a_{14}\right]\right]\right],\left[\left[\left[a_{4}, a_{12}\right],\left[a_{6}, a_{14}\right]\right],\left[\left[a_{5}, a_{13}\right],\left[a_{7}\right.\right.\right.\right.$, $\left.\left.\left.\left.\mathrm{a}_{15}\right]\right]\right]\right]=\left[\left[\mathrm{a}_{3}, \mathrm{a}_{11}\right],\left[\mathrm{a}_{7}, \mathrm{a}_{15}\right]\right]$

$\left[\left[\left[\left[a_{4}, a_{12}\right],\left[a_{6}, a_{14}\right]\right],\left[\left[a_{5}, a_{13}\right],\left[a_{7}, a_{15}\right]\right]\right],\left[\left[\left[a_{5}, a_{13}\right],\left[a_{7}, a_{15}\right]\right],\left[\left[a_{6}, a_{14}\right],\left[a_{8}\right.\right.\right.\right.$, $\left.\left.\left.\left.\mathrm{a}_{16}\right]\right]\right]\right]=\left[\left[\mathrm{a}_{4}, \mathrm{a}_{12}\right],\left[\mathrm{a}_{8}, \mathrm{a}_{16}\right]\right]$ 
[[[[a $\left.\left.\left.\left.a_{5}, a_{13}\right],\left[a_{7}, a_{15}\right]\right],\left[\left[a_{6}, a_{14}\right],\left[a_{8}, a_{16}\right]\right]\right],\left[\left[\left[a_{6}, a_{14}\right],\left[a_{8}, a_{16}\right]\right],\left[\left[a_{7}, a_{15}\right],\left[a_{9}, a_{1}\right]\right]\right]\right]=\left[\left[a_{5}\right.\right.$, $\left.\left.\mathrm{a}_{13}\right],\left[\mathrm{a}_{9}, \mathrm{a}_{1}\right]\right]=\left[\left[\mathrm{a}_{1}, \mathrm{a}_{9}\right],\left[\mathrm{a}_{5}, \mathrm{a}_{13}\right]\right]$

$\left[\left[\left[\left[a_{6}, a_{14}\right],\left[a_{8}, a_{16}\right]\right],\left[\left[a_{7}, a_{15}\right],\left[a_{9}, a_{1}\right]\right]\right],\left[\left[\left[a_{7}, a_{15}\right],\left[a_{9}, a_{1}\right]\right],\left[\left[a_{8}, a_{16}\right],\left[a_{10}, a_{2}\right]\right]\right]\right]=\left[\left[a_{6}\right.\right.$, $\left.\left.\mathrm{a}_{14}\right],\left[\mathrm{a}_{10}, \mathrm{a}_{2}\right]\right]=\left[\left[\mathrm{a}_{2}, \mathrm{a}_{10}\right],\left[\mathrm{a}_{6}, \mathrm{a}_{14}\right]\right]$

[[[[a $\left.\left.\left.\left.a_{7}, a_{15}\right],\left[a_{9}, a_{1}\right]\right],\left[\left[a_{8}, a_{16}\right],\left[a_{10}, a_{2}\right]\right]\right],\left[\left[\left[a_{8}, a_{16}\right],\left[a_{10}, a_{2}\right]\right],\left[\left[a_{9}, a_{1}\right],\left[a_{11}, a_{3}\right]\right]\right]\right]=\left[\left[a_{7}\right.\right.$, $\left.\left.a_{15}\right],\left[a_{11}, a_{3}\right]\right]=\left[\left[a_{3}, a_{11}\right],\left[a_{7}, a_{15}\right]\right]$

$\left[\left[\left[\left[a_{8}, a_{16}\right],\left[a_{10}, a_{2}\right]\right],\left[\left[a_{9}, a_{1}\right],\left[a_{11}, a_{3}\right]\right]\right],\left[\left[\left[a_{9}, a_{1}\right],\left[a_{11}, a_{3}\right]\right],\left[\left[a_{10}, a_{2}\right],\left[a_{12}, a_{4}\right]\right]\right]\right]=\left[\left[a_{8}\right.\right.$, $\left.\left.\mathrm{a}_{16}\right],\left[\mathrm{a}_{12}, \mathrm{a}_{4}\right]\right]=\left[\left[\mathrm{a}_{4}, \mathrm{a}_{12}\right],\left[\mathrm{a}_{8}, \mathrm{a}_{16}\right]\right]$

$\left[\left[\left[\left[a_{9}, a_{1}\right],\left[a_{11}, a_{3}\right]\right],\left[\left[a_{10}, a_{2}\right],\left[a_{12}, a_{4}\right]\right]\right],\left[\left[\left[a_{10}, a_{2}\right],\left[a_{12}, a_{4}\right]\right],\left[\left[a_{11}, a_{3}\right],\left[a_{13}, a_{5}\right]\right]\right]=\left[\left[a_{9}\right.\right.\right.$, $\left.\left.a_{1}\right],\left[a_{13}, a_{5}\right]\right]=\left[\left[a_{1}, a_{9}\right],\left[a_{5}, a_{13}\right]\right]$

$\left[\left[\left[\left[a_{10}, a_{2}\right],\left[a_{12}, a_{4}\right]\right],\left[\left[a_{11}, a_{3}\right],\left[a_{13}, a_{5}\right]\right]\right],\left[\left[\left[a_{11}, a_{3}\right],\left[a_{13}, a_{5}\right]\right],\left[\left[a_{12}, a_{4}\right],\left[a_{14}\right.\right.\right.\right.$, $\left.\left.\left.\left.\mathrm{a}_{6}\right]\right]\right]\right]=\left[\left[\mathrm{a}_{10}, \mathrm{a}_{2}\right],\left[\mathrm{a}_{14}, \mathrm{a}_{6}\right]\right]=\left[\left[\mathrm{a}_{2}, \mathrm{a}_{10}\right],\left[\mathrm{a}_{6}, \mathrm{a}_{14}\right]\right]$

$\left[\left[\left[\left[a_{11}, a_{3}\right],\left[a_{13}, a_{5}\right]\right],\left[\left[a_{12}, a_{4}\right],\left[a_{14}, a_{6}\right]\right]\right],\left[\left[\left[a_{12}, a_{4}\right],\left[a_{14}, a_{6}\right]\right],\left[\left[a_{13}, a_{5}\right],\left[a_{15}\right.\right.\right.\right.$, $\left.\left.\left.\left.\mathrm{a}_{7}\right]\right]\right]\right]=\left[\left[\mathrm{a}_{11}, \mathrm{a}_{3}\right],\left[\mathrm{a}_{15}, \mathrm{a}_{7}\right]\right]=\left[\left[\mathrm{a}_{3}, \mathrm{a}_{11}\right],\left[\mathrm{a}_{7}, \mathrm{a}_{15}\right]\right]$

$\left[\left[\left[\left[a_{12}, a_{4}\right],\left[a_{14}, a_{6}\right]\right],\left[\left[a_{13}, a_{5}\right],\left[a_{15}, a_{7}\right]\right]\right],\left[\left[\left[a_{13}, a_{5}\right],\left[a_{15}, a_{7}\right]\right],\left[\left[a_{14}, a_{6}\right],\left[a_{16}\right.\right.\right.\right.$, $\left.\left.\left.\left.\mathrm{a}_{8}\right]\right]\right]\right]=\left[\left[\mathrm{a}_{12}, \mathrm{a}_{4}\right],\left[\mathrm{a}_{16}, \mathrm{a}_{8}\right]\right]=\left[\left[\mathrm{a}_{4}, \mathrm{a}_{12}\right],\left[\mathrm{a}_{8}, \mathrm{a}_{16}\right]\right]$

$\left[\left[\left[\left[a_{13}, a_{5}\right],\left[a_{15}, a_{7}\right]\right],\left[\left[a_{14}, a_{6}\right],\left[a_{16}, a_{8}\right]\right]\right],\left[\left[\left[a_{14}, a_{6}\right],\left[a_{16}, a_{8}\right]\right],\left[\left[a_{15}, a_{7}\right],\left[a_{1}\right.\right.\right.\right.$, $\left.\left.\left.\left.\mathrm{a}_{9}\right]\right]\right]\right]=\left[\left[\mathrm{a}_{13}, \mathrm{a}_{5}\right],\left[\mathrm{a}_{1}, \mathrm{a}_{9}\right]\right]=\left[\left[\mathrm{a}_{1}, \mathrm{a}_{9}\right],\left[\mathrm{a}_{5}, \mathrm{a}_{13}\right]\right]$

$\left[\left[\left[\left[a_{14}, a_{6}\right],\left[a_{16}, a_{8}\right]\right],\left[\left[a_{15}, a_{7}\right],\left[a_{1}, a_{9}\right]\right]\right],\left[\left[\left[a_{15}, a_{7}\right],\left[a_{1}, a_{9}\right]\right],\left[\left[a_{16}, a_{8}\right],\left[a_{2}, a_{10}\right]\right]\right]\right]=\left[\left[a_{14}\right.\right.$, $\left.\left.\mathrm{a}_{6}\right],\left[\mathrm{a}_{2}, \mathrm{a}_{10}\right]\right]=\left[\left[\mathrm{a}_{2}, \mathrm{a}_{10}\right],\left[\mathrm{a}_{6}, \mathrm{a}_{14}\right]\right]$

[[[[a $\left.\left.\left.\left.a_{15}, a_{7}\right],\left[a_{1}, a_{9}\right]\right],\left[\left[a_{16}, a_{8}\right],\left[a_{2}, a_{10}\right]\right]\right],\left[\left[\left[a_{16}, a_{8}\right],\left[a_{2}, a_{10}\right]\right],\left[\left[a_{1}, a_{9}\right],\left[a_{3}, a_{11}\right]\right]\right]\right]=\left[\left[a_{15}\right.\right.$, $\left.\left.\mathrm{a}_{7}\right],\left[\mathrm{a}_{3}, \mathrm{a}_{11}\right]\right]=\left[\left[\mathrm{a}_{3}, \mathrm{a}_{11}\right],\left[\mathrm{a}_{7}, \mathrm{a}_{15}\right]\right]$

[[[[a $\left.\left.\left.\left.a_{16}, a_{8}\right],\left[a_{2}, a_{10}\right]\right],\left[\left[a_{1}, a_{9}\right],\left[a_{3}, a_{11}\right]\right]\right],\left[\left[\left[a_{1}, a_{9}\right],\left[a_{3}, a_{11}\right]\right],\left[\left[a_{2}, a_{10}\right],\left[a_{4}, a_{12}\right]\right]\right]\right]=\left[\left[a_{16}\right.\right.$, $\left.\left.\mathrm{a}_{8}\right],\left[\mathrm{a}_{4}, \mathrm{a}_{12}\right]\right]=\left[\left[\mathrm{a}_{4}, \mathrm{a}_{12}\right],\left[\mathrm{a}_{8}, \mathrm{a}_{16}\right]\right]$

Step13

$\left[\left[\left[a_{1}, a_{9}\right],\left[a_{5}, a_{13}\right]\right],\left[\left[a_{2}, a_{10}\right],\left[a_{6}, a_{14}\right]\right]\right]$

$\left[\left[\left[a_{2}, a_{10}\right],\left[a_{6}, a_{14}\right]\right],\left[\left[a_{3}, a_{11}\right],\left[a_{7}, a_{15}\right]\right]\right]$

$\left[\left[\left[\mathrm{a}_{3}, \mathrm{a}_{11}\right],\left[\mathrm{a}_{7}, \mathrm{a}_{15}\right]\right],\left[\left[\mathrm{a}_{4}, \mathrm{a}_{12}\right],\left[\mathrm{a}_{8}, \mathrm{a}_{16}\right]\right]\right]$

$\left[\left[\left[a_{4}, a_{12}\right],\left[a_{8}, a_{16}\right]\right],\left[\left[a_{1}, a_{9}\right],\left[a_{5}, a_{13}\right]\right]\right]$

$\left[\left[\left[a_{1}, a_{9}\right],\left[a_{5}, a_{13}\right]\right],\left[\left[a_{2}, a_{10}\right],\left[a_{6}, a_{14}\right]\right]\right]$

$\left[\left[\left[\mathrm{a}_{2}, \mathrm{a}_{10}\right],\left[\mathrm{a}_{6}, \mathrm{a}_{14}\right]\right],\left[\left[\mathrm{a}_{3}, \mathrm{a}_{11}\right],\left[\mathrm{a}_{7}, \mathrm{a}_{15}\right]\right]\right]$

$\left[\left[\left[\mathrm{a}_{3}, \mathrm{a}_{11}\right],\left[\mathrm{a}_{7}, \mathrm{a}_{15}\right]\right],\left[\left[\mathrm{a}_{4}, \mathrm{a}_{12}\right],\left[\mathrm{a}_{8}, \mathrm{a}_{16}\right]\right]\right]$

$\left[\left[\left[a_{4}, a_{12}\right],\left[a_{8}, a_{16}\right]\right],\left[\left[a_{1}, a_{9}\right],\left[a_{5}, a_{13}\right]\right]\right]$

$\left[\left[\left[\mathrm{a}_{1}, \mathrm{a}_{9}\right],\left[\mathrm{a}_{5}, \mathrm{a}_{13}\right]\right],\left[\left[\mathrm{a}_{2}, \mathrm{a}_{10}\right],\left[\mathrm{a}_{6}, \mathrm{a}_{14}\right]\right]\right]$

$\left[\left[\left[\mathrm{a}_{2}, \mathrm{a}_{10}\right],\left[\mathrm{a}_{6}, \mathrm{a}_{14}\right]\right],\left[\left[\mathrm{a}_{3}, \mathrm{a}_{11}\right],\left[\mathrm{a}_{7}, \mathrm{a}_{15}\right]\right]\right]$

$\left[\left[\left[\mathrm{a}_{3}, \mathrm{a}_{11}\right],\left[\mathrm{a}_{7}, \mathrm{a}_{15}\right]\right],\left[\left[\mathrm{a}_{4}, \mathrm{a}_{12}\right],\left[\mathrm{a}_{8}, \mathrm{a}_{16}\right]\right]\right]$

$\left[\left[\left[a_{4}, a_{12}\right],\left[a_{8}, a_{16}\right]\right] .\left[\left[a_{1}, a_{9}\right],\left[a_{5}, a_{13}\right]\right]\right]$

$\left[\left[\left[a_{1}, a_{9}\right],\left[a_{5}, a_{13}\right]\right],\left[\left[a_{2}, a_{10}\right],\left[a_{6}, a_{14}\right]\right]\right]$

$\left[\left[\left[a_{2}, a_{10}\right],\left[a_{6}, a_{14}\right]\right],\left[\left[a_{3}, a_{11}\right],\left[a_{7}, a_{15}\right]\right]\right]$

$\left[\left[\left[\mathrm{a}_{3}, \mathrm{a}_{11}\right],\left[\mathrm{a}_{7}, \mathrm{a}_{15}\right]\right],\left[\left[\mathrm{a}_{4}, \mathrm{a}_{12}\right],\left[\mathrm{a}_{8}, \mathrm{a}_{16}\right]\right]\right]$

$\left[\left[\left[\mathrm{a}_{4}, \mathrm{a}_{12}\right],\left[\mathrm{a}_{8}, \mathrm{a}_{16}\right]\right],\left[\left[\mathrm{a}_{1}, \mathrm{a}_{9}\right],\left[\mathrm{a}_{5}, \mathrm{a}_{13}\right]\right]\right]$

Step14 (Remark: any integer at vertices follows the case iii) of lemma 2.1.)

$\left[\left[\left[\left[\mathrm{a}_{1}, \mathrm{a}_{9}\right],\left[\mathrm{a}_{5}, \mathrm{a}_{13}\right]\right],\left[\left[\mathrm{a}_{2}, \mathrm{a}_{10}\right],\left[\mathrm{a}_{6}, \mathrm{a}_{14}\right]\right]\right],\left[\left[\left[\mathrm{a}_{2}, \mathrm{a}_{10}\right],\left[\mathrm{a}_{6}, \mathrm{a}_{14}\right]\right],\left[\left[\mathrm{a}_{3}, \mathrm{a}_{11}\right],\left[\mathrm{a}_{7}, \mathrm{a}_{15}\right]\right]\right]\right]=$ $\left[\left[\left[a_{1}, a_{9}\right],\left[a_{5}, a_{13}\right]\right],\left[\left[a_{3}, a_{11}\right],\left[a_{7}, a_{15}\right]\right]\right]$

$\left[\left[\left[\left[a_{2}, a_{10}\right],\left[a_{6}, a_{14}\right]\right],\left[\left[a_{3}, a_{11}\right],\left[a_{7}, a_{15}\right]\right]\right],\left[\left[\left[a_{3}, a_{11}\right],\left[a_{7}, a_{15}\right]\right],\left[\left[a_{4}, a_{12}\right],\left[a_{8}\right.\right.\right.\right.$, 
$\left.\left.\left.\left.\mathrm{a}_{16}\right]\right]\right]\right]=\left[\left[\left[\mathrm{a}_{2}, \mathrm{a}_{10}\right],\left[\mathrm{a}_{6}, \mathrm{a}_{14}\right]\right],\left[\left[\mathrm{a}_{4}, \mathrm{a}_{12}\right],\left[\mathrm{a}_{8}, \mathrm{a}_{16}\right]\right]\right]$

$\left[\left[\left[\left[a_{3}, a_{11}\right],\left[a_{7}, a_{15}\right]\right],\left[\left[a_{4}, a_{12}\right],\left[a_{8}, a_{16}\right]\right]\right],\left[\left[\left[a_{4}, a_{12}\right],\left[a_{8}, a_{16}\right]\right],\left[\left[a_{1}, a_{9}\right],\left[a_{5}, a_{13}\right]\right]\right]\right]=$ $\left[\left[\left[a_{3}, a_{11}\right],\left[a_{7}, a_{15}\right]\right],\left[\left[a_{1}, a_{9}\right],\left[a_{5}, a_{13}\right]\right]\right]$

$=\left[\left[\left[a_{1}, a_{3}\right],\left[a_{5}, a_{13}\right]\right],\left[\left[a_{3}, a_{11}\right],\left[a_{7}, a_{15}\right]\right]\right]$

$\left[\left[\left[\left[a_{4}, a_{12}\right],\left[a_{8}, a_{16}\right]\right],\left[\left[a_{1}, a_{9}\right],\left[a_{5}, a_{13}\right]\right]\right],\left[\left[\left[a_{1}, a_{9}\right],\left[a_{5}, a_{13}\right]\right],\left[\left[a_{2}, a_{10}\right],\left[a_{6}, a_{14}\right]\right]\right]\right]=$ $\left[\left[\left[a_{4}, a_{12}\right],\left[a_{8}, a_{16}\right]\right],\left[\left[a_{2}, a_{10}\right],\left[a_{6}, a_{14}\right]\right]\right]$

$=\left[\left[\left[\mathrm{a}_{2}, \mathrm{a}_{10}\right],\left[\mathrm{a}_{6}, \mathrm{a}_{14}\right]\right],\left[\left[\mathrm{a}_{4}, \mathrm{a}_{12}\right],\left[\mathrm{a}_{8}, \mathrm{a}_{16}\right]\right]\right]$

$\left[\left[\left[\left[a_{1}, a_{9}\right],\left[a_{5}, a_{13}\right]\right],\left[\left[a_{2}, a_{10}\right],\left[a_{6}, a_{14}\right]\right]\right],\left[\left[\left[a_{2}, a_{10}\right],\left[a_{6}, a_{14}\right]\right],\left[\left[a_{3}, a_{11}\right],\left[a_{7}\right.\right.\right.\right.$, $\left.\left.\left.\left.\mathrm{a}_{15}\right]\right]\right]\right]=\left[\left[\left[\mathrm{a}_{1}, \mathrm{a}_{9}\right],\left[\mathrm{a}_{5}, \mathrm{a}_{13}\right]\right],\left[\left[\mathrm{a}_{3}, \mathrm{a}_{11}\right],\left[\mathrm{a}_{7}, \mathrm{a}_{15}\right]\right]\right]$

$\left[\left[\left[\left[a_{2}, a_{10}\right],\left[a_{6}, a_{14}\right]\right],\left[\left[a_{3}, a_{11}\right],\left[a_{7}, a_{15}\right]\right]\right],\left[\left[\left[a_{3}, a_{11}\right],\left[a_{7}, a_{15}\right]\right],\left[\left[a_{4}, a_{12}\right],\left[a_{8}\right.\right.\right.\right.$, $\left.\left.\left.\left.\mathrm{a}_{16}\right]\right]\right]\right]=\left[\left[\left[\mathrm{a}_{2}, \mathrm{a}_{10}\right],\left[\mathrm{a}_{6}, \mathrm{a}_{14}\right]\right],\left[\left[\mathrm{a}_{4}, \mathrm{a}_{12}\right],\left[\mathrm{a}_{8}, \mathrm{a}_{16}\right]\right]\right]$

$\left[\left[\left[\left[a_{3}, a_{11}\right],\left[a_{7}, a_{15}\right]\right],\left[\left[a_{4}, a_{12}\right],\left[a_{8}, a_{16}\right]\right],\left[\left[\left[a_{4}, a_{12}\right],\left[a_{8}, a_{16}\right]\right],\left[\left[a_{1}, a_{9}\right],\left[a_{5}\right.\right.\right.\right.\right.$, $\left.\left.\left.\left.\mathrm{a}_{13}\right]\right]\right]\right]=\left[\left[\left[\left[\mathrm{a}_{1}, \mathrm{a}_{3}\right],\left[\mathrm{a}_{5}, \mathrm{a}_{13}\right]\right],\left[\left[\mathrm{a}_{3}, \mathrm{a}_{11}\right],\left[\mathrm{a}_{7}, \mathrm{a}_{15}\right]\right]\right]\right.$

$\left[\left[\left[\left[a_{4}, a_{12}\right],\left[a_{8}, a_{16}\right]\right],\left[\left[a_{1}, a_{9}\right],\left[a_{5}, a_{13}\right]\right]\right],\left[\left[\left[a_{1}, a_{9}\right],\left[a_{5}, a_{13}\right]\right],\left[\left[a_{2}, a_{10}\right],\left[a_{6}, a_{14}\right]\right]\right]\right]=\left[\left[\left[a_{2}\right.\right.\right.$, $\left.\left.\left.\mathrm{a}_{10}\right],\left[\mathrm{a}_{6}, \mathrm{a}_{14}\right]\right],\left[\left[\mathrm{a}_{4}, \mathrm{a}_{12}\right],\left[\mathrm{a}_{8}, \mathrm{a}_{16}\right]\right]\right]$

$\left[\left[\left[\left[a_{1}, a_{9}\right],\left[a_{5}, a_{13}\right]\right],\left[\left[a_{2}, a_{10}\right],\left[a_{6}, a_{14}\right]\right]\right],\left[\left[\left[a_{2}, a_{10}\right],\left[a_{6}, a_{14}\right]\right],\left[\left[a_{3}, a_{11}\right],\left[a_{7}\right.\right.\right.\right.$, $\left.\left.\left.\mathrm{a}_{15}\right]\right]\right]=\left[\left[\left[\mathrm{a}_{1}, \mathrm{a}_{9}\right],\left[\mathrm{a}_{5}, \mathrm{a}_{13}\right]\right],\left[\left[\mathrm{a}_{3}, \mathrm{a}_{11}\right],\left[\mathrm{a}_{7}, \mathrm{a}_{15}\right]\right]\right]$

$\left[\left[\left[\left[a_{2}, a_{10}\right],\left[a_{6}, a_{14}\right]\right],\left[\left[a_{3}, a_{11}\right],\left[a_{7}, a_{15}\right]\right]\right],\left[\left[\left[a_{3}, a_{11}\right],\left[a_{7}, a_{15}\right]\right],\left[\left[a_{4}, a_{12}\right],\left[a_{8}\right.\right.\right.\right.$, $\left.\left.\left.\left.\mathrm{a}_{16}\right]\right]\right]\right]=\left[\left[\left[\mathrm{a}_{2}, \mathrm{a}_{10}\right],\left[\mathrm{a}_{6}, \mathrm{a}_{14}\right]\right],\left[\left[\mathrm{a}_{4}, \mathrm{a}_{12}\right],\left[\mathrm{a}_{8}, \mathrm{a}_{16}\right]\right]\right]$

$\left[\left[\left[\left[a_{3}, a_{11}\right],\left[a_{7}, a_{15}\right]\right],\left[\left[a_{4}, a_{12}\right],\left[a_{8}, a_{16}\right]\right]\right],\left[\left[\left[a_{4}, a_{12}\right],\left[a_{8}, a_{16}\right]\right] . \quad\left[\left[a_{1}, a_{9}\right],\left[a_{5}\right.\right.\right.\right.$, $\left.\left.\left.\left.\mathrm{a}_{13}\right]\right]\right]\right]=\left[\left[\left[\mathrm{a}_{1}, \mathrm{a}_{9}\right],\left[\mathrm{a}_{5}, \mathrm{a}_{13}\right]\right],\left[\left[\mathrm{a}_{3}, \mathrm{a}_{11}\right],\left[\mathrm{a}_{7}, \mathrm{a}_{15}\right]\right]\right]$

$\left[\left[\left[\left[a_{4}, a_{12}\right],\left[a_{8}, a_{16}\right]\right] .\left[\left[a_{1}, a_{9}\right],\left[a_{5}, a_{13}\right]\right]\right],\left[\left[\left[a_{1}, a_{9}\right],\left[a_{5}, a_{13}\right]\right],\left[\left[a_{2}, a_{10}\right],\left[a_{6}, a_{14}\right]\right]\right]\right]=\left[\left[\left[a_{2}\right.\right.\right.$, $\left.\left.\left.\mathrm{a}_{10}\right],\left[\mathrm{a}_{6}, \mathrm{a}_{14}\right]\right],\left[\left[\mathrm{a}_{4}, \mathrm{a}_{12}\right],\left[\mathrm{a}_{8}, \mathrm{a}_{16}\right]\right]\right]$

$\left[\left[\left[\left[a_{1}, a_{9}\right],\left[a_{5}, a_{13}\right]\right],\left[\left[a_{2}, a_{10}\right],\left[a_{6}, a_{14}\right]\right]\right],\left[\left[\left[a_{2}, a_{10}\right],\left[a_{6}, a_{14}\right]\right],\left[\left[a_{3}, a_{11}\right],\left[a_{7}\right.\right.\right.\right.$, $\left.\left.\left.\left.\mathrm{a}_{15}\right]\right]\right]\right]=\left[\left[\left[\mathrm{a}_{1}, \mathrm{a}_{9}\right],\left[\mathrm{a}_{5}, \mathrm{a}_{13}\right]\right],\left[\left[\mathrm{a}_{3}, \mathrm{a}_{11}\right],\left[\mathrm{a}_{7}, \mathrm{a}_{15}\right]\right]\right]$

$\left[\left[\left[\left[a_{2}, a_{10}\right],\left[a_{6}, a_{14}\right]\right],\left[\left[a_{3}, a_{11}\right],\left[a_{7}, a_{15}\right]\right]\right],\left[\left[\left[a_{3}, a_{11}\right],\left[a_{7}, a_{15}\right]\right],\left[\left[a_{4}, a_{12}\right],\left[a_{8}\right.\right.\right.\right.$, $\left.\left.\left.\left.\mathrm{a}_{16}\right]\right]\right]\right]=\left[\left[\left[\mathrm{a}_{2}, \mathrm{a}_{10}\right],\left[\mathrm{a}_{6}, \mathrm{a}_{14}\right]\right],\left[\left[\mathrm{a}_{4}, \mathrm{a}_{12}\right],\left[\mathrm{a}_{8}, \mathrm{a}_{16}\right]\right]\right]$

$\left[\left[\left[\left[a_{3}, a_{11}\right],\left[a_{7}, a_{15}\right]\right],\left[\left[a_{4}, a_{12}\right],\left[a_{8}, a_{16}\right]\right]\right],\left[\left[\left[a_{4}, a_{12}\right],\left[a_{8}, a_{16}\right]\right],\left[\left[a_{1}, a_{9}\right],\left[a_{5}\right.\right.\right.\right.$, $\left.\left.\left.\left.\mathrm{a}_{13}\right]\right]\right]\right]=\left[\left[\left[\mathrm{a}_{1}, \mathrm{a}_{9}\right],\left[\mathrm{a}_{5}, \mathrm{a}_{13}\right]\right],\left[\left[\mathrm{a}_{3}, \mathrm{a}_{11}\right],\left[\mathrm{a}_{7}, \mathrm{a}_{15}\right]\right]\right]$

[[[[a $\left.\left.\left.\left.a_{4}, a_{12}\right],\left[a_{8}, a_{16}\right]\right],\left[\left[a_{1}, a_{9}\right],\left[a_{5}, a_{13}\right]\right]\right],\left[\left[\left[a_{1}, a_{9}\right],\left[a_{5}, a_{13}\right]\right],\left[\left[a_{2}, a_{10}\right],\left[a_{6}, a_{14}\right]\right]\right]\right]=\left[\left[\left[a_{2}\right.\right.\right.$, $\left.\left.\left.\mathrm{a}_{10}\right],\left[\mathrm{a}_{6}, \mathrm{a}_{14}\right]\right],\left[\left[\mathrm{a}_{4}, \mathrm{a}_{12}\right],\left[\mathrm{a}_{8}, \mathrm{a}_{16}\right]\right]\right]$

Step15

[[[[a $\left.\left.\left.a_{1}, a_{9}\right],\left[a_{5}, a_{13}\right]\right],\left[\left[a_{3}, a_{11}\right],\left[a_{7}, a_{15}\right]\right]\right],\left[\left[\left[a_{2}, a_{10}\right],\left[a_{6}, a_{14}\right]\right],\left[\left[a_{4}, a_{12}\right],\left[a_{8}, a_{16}\right]\right]\right]$ $\left[\left[\left[\left[a_{2}, a_{10}\right],\left[a_{6}, a_{14}\right]\right],\left[\left[a_{4}, a_{12}\right],\left[a_{8}, a_{16}\right]\right]\right],\left[\left[\left[a_{1}, a_{9}\right],\left[a_{5}, a_{13}\right]\right],\left[\left[a_{3}, a_{11}\right],\left[a_{7}, a_{15}\right]\right]\right]\right.$ $=\left[\left[\left[\left[\mathrm{a}_{1}, \mathrm{a}_{9}\right],\left[\mathrm{a}_{5}, \mathrm{a}_{13}\right]\right],\left[\left[\mathrm{a}_{3}, \mathrm{a}_{11}\right],\left[\mathrm{a}_{7}, \mathrm{a}_{15}\right]\right]\right],\left[\left[\left[\mathrm{a}_{2}, \mathrm{a}_{10}\right],\left[\mathrm{a}_{6}, \mathrm{a}_{14}\right]\right],\left[\left[\mathrm{a}_{4}, \mathrm{a}_{12}\right],\left[\mathrm{a}_{8}, \mathrm{a}_{16}\right]\right]\right]\right]$ $\left[\left[\left[\left[\mathrm{a}_{1}, \mathrm{a}_{9}\right],\left[\mathrm{a}_{5}, \mathrm{a}_{13}\right]\right],\left[\left[\mathrm{a}_{3}, \mathrm{a}_{11}\right],\left[\mathrm{a}_{7}, \mathrm{a}_{15}\right]\right]\right],\left[\left[\left[\mathrm{a}_{2}, \mathrm{a}_{10}\right],\left[\mathrm{a}_{6}, \mathrm{a}_{14}\right]\right],\left[\left[\mathrm{a}_{4}, \mathrm{a}_{12}\right],\left[\mathrm{a}_{8}, \mathrm{a}_{16}\right]\right]\right]\right]$ $\left[\left[\left[\left[\mathrm{a}_{2}, \mathrm{a}_{10}\right],\left[\mathrm{a}_{6}, \mathrm{a}_{14}\right]\right],\left[\left[\mathrm{a}_{4}, \mathrm{a}_{12}\right],\left[\mathrm{a}_{8}, \mathrm{a}_{16}\right]\right]\right],\left[\left[\left[\mathrm{a}_{1}, \mathrm{a}_{9}\right],\left[\mathrm{a}_{5}, \mathrm{a}_{13}\right]\right],\left[\left[\mathrm{a}_{3}, \mathrm{a}_{11}\right],\left[\mathrm{a}_{7}, \mathrm{a}_{15}\right]\right]\right]\right]$ $=\left[\left[\left[\left[\mathrm{a}_{1}, \mathrm{a}_{9}\right],\left[\mathrm{a}_{5}, \mathrm{a}_{13}\right]\right],\left[\left[\mathrm{a}_{3}, \mathrm{a}_{11}\right],\left[\mathrm{a}_{7}, \mathrm{a}_{15}\right]\right]\right],\left[\left[\left[\mathrm{a}_{2}, \mathrm{a}_{10}\right],\left[\mathrm{a}_{6}, \mathrm{a}_{14}\right]\right],\left[\left[\mathrm{a}_{4}, \mathrm{a}_{12}\right],\left[\mathrm{a}_{8}, \mathrm{a}_{16}\right]\right]\right]\right]$ $\left[\left[\left[\left[a_{1}, a_{9}\right],\left[a_{5}, a_{13}\right]\right],\left[\left[a_{3}, a_{11}\right],\left[a_{7}, a_{15}\right]\right]\right],\left[\left[\left[a_{2}, a_{10}\right],\left[a_{6}, a_{14}\right]\right],\left[\left[a_{4}, a_{12}\right],\left[a_{8}, a_{16}\right]\right]\right]\right.$ $\left[\left[\left[\left[\mathrm{a}_{2}, \mathrm{a}_{10}\right],\left[\mathrm{a}_{6}, \mathrm{a}_{14}\right]\right],\left[\left[\mathrm{a}_{4}, \mathrm{a}_{12}\right],\left[\mathrm{a}_{8}, \mathrm{a}_{16}\right]\right]\right],\left[\left[\left[\mathrm{a}_{1}, \mathrm{a}_{9}\right],\left[\mathrm{a}_{5}, \mathrm{a}_{13}\right]\right],\left[\left[\mathrm{a}_{3}, \mathrm{a}_{11}\right],\left[\mathrm{a}_{7}, \mathrm{a}_{15}\right]\right]\right]\right]$ $=\left[\left[\left[\left[a_{1}, a_{9}\right],\left[a_{5}, a_{13}\right]\right],\left[\left[a_{3}, a_{11}\right],\left[a_{7}, a_{15}\right]\right]\right],\left[\left[\left[a_{2}, a_{10}\right],\left[a_{6}, a_{14}\right]\right],\left[\left[a_{4}, a_{12}\right],\left[a_{8}, a_{16}\right]\right]\right]\right]$ $\left[\left[\left[\left[\mathrm{a}_{1}, \mathrm{a}_{9}\right],\left[\mathrm{a}_{5}, \mathrm{a}_{13}\right]\right],\left[\left[\mathrm{a}_{3}, \mathrm{a}_{11}\right],\left[\mathrm{a}_{7}, \mathrm{a}_{15}\right]\right]\right],\left[\left[\left[\mathrm{a}_{2}, \mathrm{a}_{10}\right],\left[\mathrm{a}_{6}, \mathrm{a}_{14}\right]\right],\left[\left[\mathrm{a}_{4}, \mathrm{a}_{12}\right],\left[\mathrm{a}_{8}, \mathrm{a}_{16}\right]\right]\right]\right]$ $\left[\left[\left[\left[\mathrm{a}_{2}, \mathrm{a}_{10}\right],\left[\mathrm{a}_{6}, \mathrm{a}_{14}\right]\right],\left[\left[\mathrm{a}_{4}, \mathrm{a}_{12}\right],\left[\mathrm{a}_{8}, \mathrm{a}_{16}\right]\right]\right],\left[\left[\left[\mathrm{a}_{1}, \mathrm{a}_{9}\right],\left[\mathrm{a}_{5}, \mathrm{a}_{13}\right]\right],\left[\left[\mathrm{a}_{3}, \mathrm{a}_{11}\right],\left[\mathrm{a}_{7}, \mathrm{a}_{15}\right]\right]\right]\right]$ $=\left[\left[\left[\left[\mathrm{a}_{1}, \mathrm{a}_{9}\right],\left[\mathrm{a}_{5}, \mathrm{a}_{13}\right]\right],\left[\left[\mathrm{a}_{3}, \mathrm{a}_{11}\right],\left[\mathrm{a}_{7}, \mathrm{a}_{15}\right]\right]\right],\left[\left[\left[\mathrm{a}_{2}, \mathrm{a}_{10}\right],\left[\mathrm{a}_{6}, \mathrm{a}_{14}\right]\right],\left[\left[\mathrm{a}_{4}, \mathrm{a}_{12}\right],\left[\mathrm{a}_{8}, \mathrm{a}_{16}\right]\right]\right]\right]$ $\left[\left[\left[\left[\mathrm{a}_{1}, \mathrm{a}_{9}\right],\left[\mathrm{a}_{5}, \mathrm{a}_{13}\right]\right],\left[\left[\mathrm{a}_{3}, \mathrm{a}_{11}\right],\left[\mathrm{a}_{7}, \mathrm{a}_{15}\right]\right]\right],\left[\left[\left[\mathrm{a}_{2}, \mathrm{a}_{10}\right],\left[\mathrm{a}_{6}, \mathrm{a}_{14}\right]\right],\left[\left[\mathrm{a}_{4}, \mathrm{a}_{12}\right],\left[\mathrm{a}_{8}, \mathrm{a}_{16}\right]\right]\right]\right]$ 
[[[[a $\left.\left.\left.\left.\mathrm{a}_{2}, \mathrm{a}_{10}\right],\left[\mathrm{a}_{6}, \mathrm{a}_{14}\right]\right],\left[\left[\mathrm{a}_{4}, \mathrm{a}_{12}\right],\left[\mathrm{a}_{8}, \mathrm{a}_{16}\right]\right]\right],\left[\left[\left[\mathrm{a}_{1}, \mathrm{a}_{9}\right],\left[\mathrm{a}_{5}, \mathrm{a}_{13}\right]\right],\left[\left[\mathrm{a}_{3}, \mathrm{a}_{11}\right],\left[\mathrm{a}_{7}, \mathrm{a}_{15}\right]\right]\right]\right]$ $=\left[\left[\left[\left[\mathrm{a}_{1}, \mathrm{a}_{9}\right],\left[\mathrm{a}_{5}, \mathrm{a}_{13}\right]\right],\left[\left[\mathrm{a}_{3}, \mathrm{a}_{11}\right],\left[\mathrm{a}_{7}, \mathrm{a}_{15}\right]\right]\right],\left[\left[\left[\mathrm{a}_{2}, \mathrm{a}_{10}\right],\left[\mathrm{a}_{6}, \mathrm{a}_{14}\right]\right],\left[\left[\mathrm{a}_{4}, \mathrm{a}_{12}\right],\left[\mathrm{a}_{8}, \mathrm{a}_{16}\right]\right]\right]\right]$ $\left[\left[\left[\left[\mathrm{a}_{1}, \mathrm{a}_{9}\right],\left[\mathrm{a}_{5}, \mathrm{a}_{13}\right]\right],\left[\left[\mathrm{a}_{3}, \mathrm{a}_{11}\right],\left[\mathrm{a}_{7}, \mathrm{a}_{15}\right]\right]\right],\left[\left[\left[\mathrm{a}_{2}, \mathrm{a}_{10}\right],\left[\mathrm{a}_{6}, \mathrm{a}_{14}\right]\right],\left[\left[\mathrm{a}_{4}, \mathrm{a}_{12}\right],\left[\mathrm{a}_{8}, \mathrm{a}_{16}\right]\right]\right]\right]$ $\left[\left[\left[\left[a_{2}, a_{10}\right],\left[a_{6}, a_{14}\right]\right],\left[\left[a_{4}, a_{12}\right],\left[a_{8}, a_{16}\right]\right]\right],\left[\left[\left[a_{1}, a_{9}\right],\left[a_{5}, a_{13}\right]\right],\left[\left[a_{3}, a_{11}\right],\left[a_{7}, a_{15}\right]\right]\right]\right]$ $=\left[\left[\left[\left[\mathrm{a}_{1}, \mathrm{a}_{9}\right],\left[\mathrm{a}_{5}, \mathrm{a}_{13}\right]\right],\left[\left[\mathrm{a}_{3}, \mathrm{a}_{11}\right],\left[\mathrm{a}_{7}, \mathrm{a}_{15}\right]\right]\right],\left[\left[\left[\mathrm{a}_{2}, \mathrm{a}_{10}\right],\left[\mathrm{a}_{6}, \mathrm{a}_{14}\right]\right],\left[\left[\mathrm{a}_{4}, \mathrm{a}_{12}\right],\left[\mathrm{a}_{8}, \mathrm{a}_{16}\right]\right]\right]\right]$ $\left[\left[\left[\left[a_{1}, a_{9}\right],\left[a_{5}, a_{13}\right]\right],\left[\left[a_{3}, a_{11}\right],\left[a_{7}, a_{15}\right]\right]\right],\left[\left[\left[a_{2}, a_{10}\right],\left[a_{6}, a_{14}\right]\right],\left[\left[a_{4}, a_{12}\right],\left[a_{8}, a_{16}\right]\right]\right]\right]$ [[[[a $\left.\left.\left.\left.\mathrm{a}_{2}, \mathrm{a}_{10}\right],\left[\mathrm{a}_{6}, \mathrm{a}_{14}\right]\right],\left[\left[\mathrm{a}_{4}, \mathrm{a}_{12}\right],\left[\mathrm{a}_{8}, \mathrm{a}_{16}\right]\right]\right],\left[\left[\left[\mathrm{a}_{1}, \mathrm{a}_{9}\right],\left[\mathrm{a}_{5}, \mathrm{a}_{13}\right]\right],\left[\left[\mathrm{a}_{3}, \mathrm{a}_{11}\right],\left[\mathrm{a}_{7}, \mathrm{a}_{15}\right]\right]\right]\right]$ $=\left[\left[\left[\left[\mathrm{a}_{1}, \mathrm{a}_{9}\right],\left[\mathrm{a}_{5}, \mathrm{a}_{13}\right]\right],\left[\left[\mathrm{a}_{3}, \mathrm{a}_{11}\right],\left[\mathrm{a}_{7}, \mathrm{a}_{15}\right]\right]\right],\left[\left[\left[\mathrm{a}_{2}, \mathrm{a}_{10}\right],\left[\mathrm{a}_{6}, \mathrm{a}_{14}\right]\right],\left[\left[\mathrm{a}_{4}, \mathrm{a}_{12}\right],\left[\mathrm{a}_{8}, \mathrm{a}_{16}\right]\right]\right]\right]$ [[[[a $\left.\left.\left.\left.\mathrm{a}_{1}, \mathrm{a}_{9}\right],\left[\mathrm{a}_{5}, \mathrm{a}_{13}\right]\right],\left[\left[\mathrm{a}_{3}, \mathrm{a}_{11}\right],\left[\mathrm{a}_{7}, \mathrm{a}_{15}\right]\right]\right],\left[\left[\left[\mathrm{a}_{2}, \mathrm{a}_{10}\right],\left[\mathrm{a}_{6}, \mathrm{a}_{14}\right]\right],\left[\left[\mathrm{a}_{4}, \mathrm{a}_{12}\right],\left[\mathrm{a}_{8}, \mathrm{a}_{16}\right]\right]\right]\right]$ [[[a $\left.\left.\left.\left.\mathrm{a}_{2}, \mathrm{a}_{10}\right],\left[\mathrm{a}_{6}, \mathrm{a}_{14}\right]\right],\left[\left[\mathrm{a}_{4}, \mathrm{a}_{12}\right],\left[\mathrm{a}_{8}, \mathrm{a}_{16}\right]\right]\right],\left[\left[\left[\mathrm{a}_{1}, \mathrm{a}_{9}\right],\left[\mathrm{a}_{5}, \mathrm{a}_{13}\right]\right],\left[\left[\mathrm{a}_{3}, \mathrm{a}_{11}\right],\left[\mathrm{a}_{7}, \mathrm{a}_{15}\right]\right]\right]\right]$ $=\left[\left[\left[\left[a_{1}, a_{9}\right],\left[a_{5}, a_{13}\right]\right],\left[\left[a_{3}, a_{11}\right],\left[a_{7}, a_{15}\right]\right]\right],\left[\left[\left[a_{2}, a_{10}\right],\left[a_{6}, a_{14}\right]\right],\left[\left[a_{4}, a_{12}\right],\left[a_{8}, a_{16}\right]\right]\right]\right]$ Step16

0 allout 